\title{
Investigation of IAQ-Relevant Surface Chemistry and Emissions on HVAC Filter Materials
}

\author{
Hugo Destaillats and William J. Fisk \\ Environmental Energy Technologies Division \\ Indoor Environment Department \\ Lawrence Berkeley National Laboratory \\ Berkeley, CA 94720
}

February 2010

This research was supported by the U.S. Environmental Protection Agency (EPA) through interagency agreement DW-89-92224401 with the U.S. Department of Energy (DOE) under Contract DE-AC02-05CH11231. 


\title{
Investigation of IAQ-Relevant Surface Chemistry and Emissions on HVAC Filter Materials
}

\author{
Authors: \\ Hugo Destaillats and William J. Fisk
}

Sponsor:

The Environmental Protection Agency (EPA)

\author{
Prepared by: \\ Lawrence Berkeley National Laboratory \\ Environmental Energy Technologies Division \\ Indoor Environment Department \\ Berkeley, CA 94720
}

Principal Investigator:

William J. Fisk

February 2010 


\section{$\underline{\text { Table of Contents }}$}

Abstract........................................... 3

A. Introduction .................................. 4

B. Methods...................................... 5

B.1. Selection, handling, deployment and

storage of HVAC filters............................. 5

B.2. Experimental setup................................ 7

B.3. Ozone breakthrough curves........................ 10

B.4. Determination of formaldehyde and

Acetaldehyde emissions............................... 10

C. Results......................................... 11

C.1. Ozone breakthrough curves............................... 11

C.2. Determination of formaldehyde and acetaldehyde emissions.................................. 20

C.3. Effect of face velocity................................... 27

C.4. Estimated impacts on indoor aldehyde concentrations............................. 29

D. Summary of major findings.................. 31

\section{Acknowledgments}

This research was supported by the U.S. Environmental Protection Agency (EPA) through interagency agreement DW-89-92224401 with the U.S. Department of Energy (DOE) under Contract DE-AC02-05CH11231. The opinions expressed do not necessarily represent the views of EPA or DOE. The authors acknowledge Gregory Brunner (EPA) and Michael Apte (LBNL) for their valuable insight and suggestions, which helped shape and improved the quality of this work. We also thank Michael Spears (LBNL) and Jérémie Almosni (ILSA-Lyon), who participated in the execution of the experimental work and data processing, as well as Richard Patterson (LBNL) and Michael Ringbom (Port of Oakland) for facilitating access to HVAC systems at their corresponding buildings for filter deployment and retrieval. Any mention of specific products or manufacturers does not constitute an endorsement or negative assessment of them. 


\section{Abstract}

Chemical reactions involving ozone of outdoor origin and indoor materials are known to be significant sources of formaldehyde and other irritant gas-phase oxidation products in the indoor environment. HVAC filters are exposed to particularly high ozone concentrations -close to outdoor levels. In this study, we investigated chemical processes taking place on the surface of filters that included fiberglass, polyester, cotton/polyester blend and synthetic (e.g., polyolefin) filter media. Ozone reactions were studied on unused filter media, and on filters that were deployed for 3 months in two different locations: at the Lawrence Berkeley National Laboratory and at the Port of Oakland. Specimens from each filter were exposed to ozone under controlled conditions in a laboratory flow tube at a constant flow of dry or humidified air ( $50 \%$ relative humidity). Ozone was generated with a UV source upstream of the flow tube, and monitored using a photometric detector. Ozone breakthrough curves were recorded for each sample exposed to $\sim 150 \mathrm{ppbv} \mathrm{O}_{3}$ for periods of $\sim 1000 \mathrm{~min}$, from which we estimated their uptake rate. Most experiments were performed at $1.3 \mathrm{~L} / \mathrm{min}$ (corresponding to a face velocity of $0.013 \mathrm{~m} / \mathrm{s}$ ), except for a few tests performed at a higher airflow rate, to obtain a face velocity of $0.093 \mathrm{~m} / \mathrm{s}$, slightly closer to HVAC operation conditions. Formaldehyde and acetaldehyde, two oxidation byproducts, were collected downstream of the filter and quantified. Emissions of these volatile aldehydes were consistently higher under humidified air than under dry conditions, at which levels were near the limit of detection. Our results confirm that there are significant reactions of ozone as air containing ozone flows through HVAC filters, particularly when the filters are loaded with particles and the air is humidified. The amount of ozone reacted was not clearly related to the types of filter media, e.g., fiberglass versus synthetic. Specific fiberglass filters that were coated with an impaction oil showed significantly higher formaldehyde emissions than most other samples. Those emissions were magnified in the presence of particles (i.e., in used filters), and were observed even in the absence of ozone, which suggests that hydrolysis of filter binder or tackifier additives may be the reason for those high emissions. Mass balance calculations indicate that the emission rates of formaldehyde and acetaldehyde from the filters are generally not large enough to substantially increase indoor formaldehyde or acetaldehyde concentrations. 


\section{A. Introduction}

The goal of this project is to develop information that will help EPA formulate its guidance with respect to building filtration in light of the recent findings from the EPA BASE study of a strong association of synthetic filters with increased reporting of building-related health symptoms (BRS) at work particularly when outdoor ozone levels are elevated. ${ }^{1}$ The more specific objective of the project is to test for a suspected chemical mechanism that would help explain these findings. A confirmation of the suspected chemical mechanism would provide a much more solid scientific foundation for guidance on filtration methods.

The findings from BASE analyses are potentially very important and suggest an easy and practical means of reducing BRS. However, those findings may be seen as controversial, and have not been replicated elsewhere, which suggest caution. Studies providing some evidence of an underlying mechanism for increased risks with synthetic filters in high ozone settings would contribute by adding physical plausibility to the purely statistical findings in the BASE study data analyses.

This research project focused on experiments to advance our current understanding of the potential formation of harmful indoor gas-phase pollutants, such as formaldehyde, in HVAC systems as air containing ozone passes through particle filters. Chemical reactions involving ozone of outdoor origin and indoor materials are known to be significant sources of formaldehyde and other irritant gas-phase oxidation products and respirable aerosol particles in the indoor environment. However, the chemical sources of many secondary indoor pollutants are poorly understood and the significance of reactions that occur on particle filters had been little studied. HVAC filters are exposed to particularly high ozone concentrations (often close to outdoor levels) and are therefore very susceptible to react with this atmospheric oxidant.

The current interim report summarizes results obtained at this stage of the project, and provides a preliminary estimation of the ozone decomposition potential and impact of aldehydes emitted by HVAC filters.

1 I. S. H. Buchanan, M. J. Mendell, A. G. Mirer, M. G. Apte. Air filter materials, outdoor ozone and building-related symptoms in the BASE study. Indoor Air 2008, 18, 144-155. 


\section{B. Methods}

\section{B.1. Selection, handling, deployment and storage of HVAC filters}

We procured a set of filters listed more frequently in the BASE study, to obtain a representative sample of those corresponding to fiberglass, polyester, cotton/polyester blends and other synthetic matrices. The complete set of filters obtained for this study is listed in Table 1. In the case of filters listed as "synthetic", the material used in one of those (Tri-Dim Tri-Pleat ES-40) is polyolefin.

Table 1: HVAC filters used in this study

\begin{tabular}{|c|c|c|c|c|}
\hline Manufacturer & Model & Panel or Roll & Media Coating & Thickness \\
\hline \multicolumn{5}{|c|}{ Fiberglass } \\
\hline $\begin{array}{c}\text { American Air Filter } \\
\text { (AAF) }\end{array}$ & $\begin{array}{c}\text { Frontline Gold Pads } \\
\text { (High Density) }\end{array}$ & Panel & $\begin{array}{l}\text { Viscosine (heavy } \\
\text { application) }\end{array}$ & $2 "$ \\
\hline American Air Filter & $\begin{array}{l}\text { Frontline Blue Pads } \\
\text { (Medium Density) }\end{array}$ & Panel & $\begin{array}{l}\text { Viscosine (medium } \\
\text { application) }\end{array}$ & $2 "$ \\
\hline American Air Filter & $\begin{array}{l}\text { Frontline Green Pads } \\
\text { (Medium Density) }\end{array}$ & Panel & $\begin{array}{l}\text { Viscosine (light } \\
\text { application) }\end{array}$ & $2 "$ \\
\hline Glasfloss & Disposable Panel & Panel & Adhesive & 2" \\
\hline $\begin{array}{c}\text { Flanders-Precision } \\
\text { aire }\end{array}$ & E-Z Flow II & Panel & Bonding resin* & $2 "$ \\
\hline \multicolumn{5}{|c|}{ Polyester } \\
\hline American Air Filter & Roll-O-Mat Green & Roll & $\begin{array}{c}\text { Viscosine (medium } \\
\text { application) }\end{array}$ & $1 "$ \\
\hline \multicolumn{5}{|c|}{ Cotton/polyester blend } \\
\hline Camfil Farr & $30 / 50$ & Panel & None & 2" \\
\hline Airguard & DP 40 & Panel & None & 2" \\
\hline \multicolumn{5}{|c|}{ Synthetic } \\
\hline Tri-Dim & Tri-Pleat ES-40 & Panel & None & 2" \\
\hline Glasfloss & Z-line ZL & Panel & $\begin{array}{l}\text { Electrostatically } \\
\text { charged }\end{array}$ & $2 "$ \\
\hline
\end{tabular}

*per manufacturer literature, the bonding resin is to provide rigidity and resistance to media compression.

A set containing each of the filter types was installed in the ventilation system of two designated buildings at LBNL (bldg 90 and bldg 46) for a period of 3 months. A few of these filters were left in those buildings for an extended period of exposure. Another set of filters was installed in the HVAC system of a building at the Port of Oakland. The choice of these two locations was due to their very different surroundings (i.e., proximity to diesel emissions by trucks and freeway traffic in the Port, and predominantly biogenic emissions at LBNL) to provide a variety of particle loading and chemical composition corresponding to outdoor air. In the case of LBNL's building 90, the HVAC system operates with a high degree 
of recirculation (higher than $50 \%$ ), while the units in building 46 do not recirculate indoor air, and therefore collected exclusively dust and particles from outdoors. The Port building also recirculated a significant fraction of indoor air. Figure 1 illustrates a subset of the deployed filters and the sampling locations.

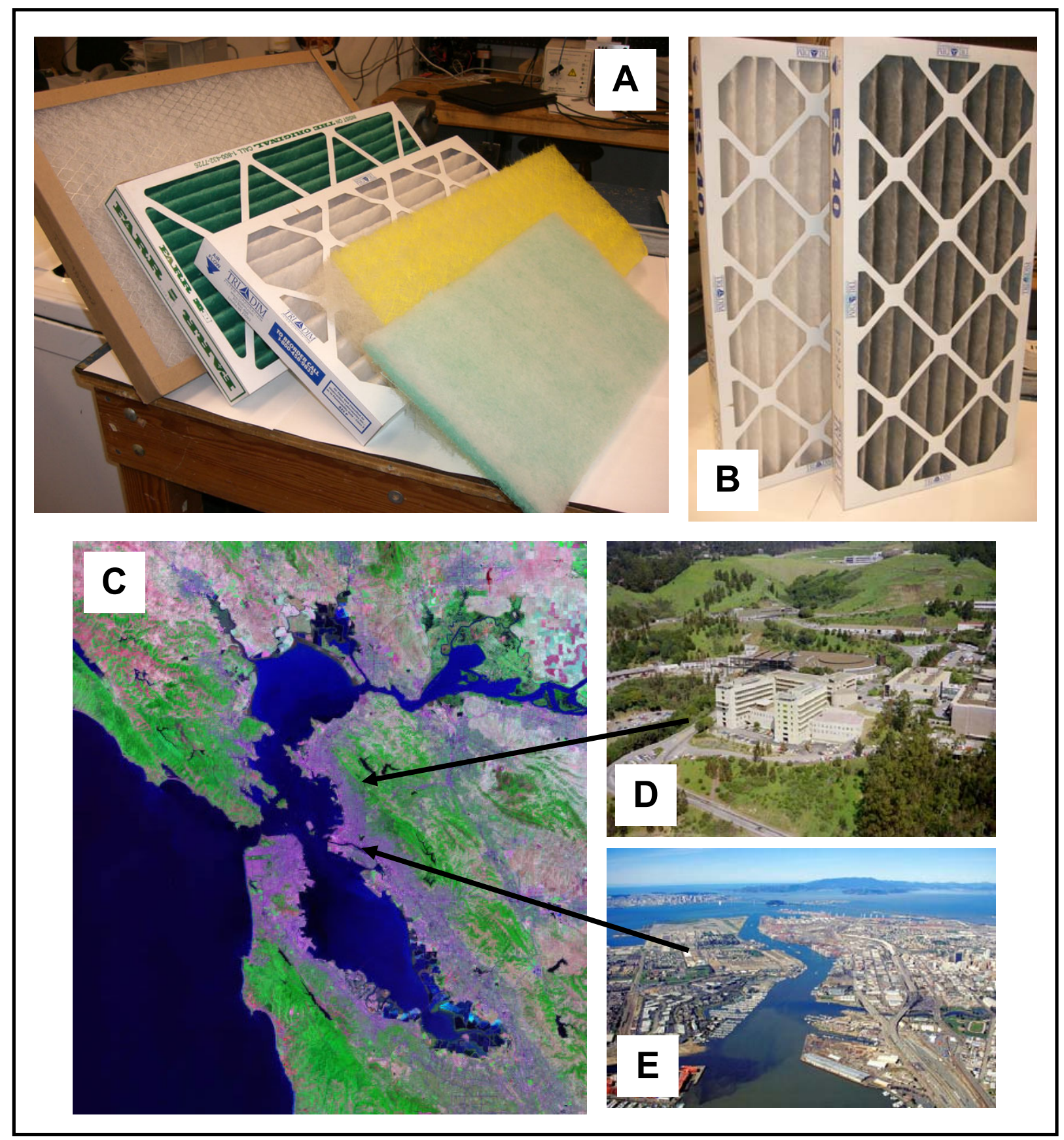

Figure 1: Deployment of filters. (A) unused filters from left to right: Glasfloss (fiberglass), Camfil Farr 30/50 (cotton/polyester), TriDim ES40 (synthetic), AAF Frontline Gold (fiberglass) and AAF Roll-O-Mat Green (polyester); (B) used and unused TriDim ES40 filters; (C) Map showing sites of deployments: (D) LBNL lab site and (E) Port of Oakland. 
In all cases, used and unused filters were wrapped with aluminum foil and stored at room temperature under $40-60 \% \mathrm{RH}$ before exposure to ozone and analysis. Specimens for each experiment consisted of $47 \mathrm{~mm}$ diameter disks that were cut from representative sections of each filter. Given the different nature and thickness of each filter material, specimens of the same cross-section had relatively different masses, some of which are illustrated in Figure 2. No significant mass changes were recorded after exposure to ozone. All specimens were used only once, and stored in a freezer at the end of each experiment.

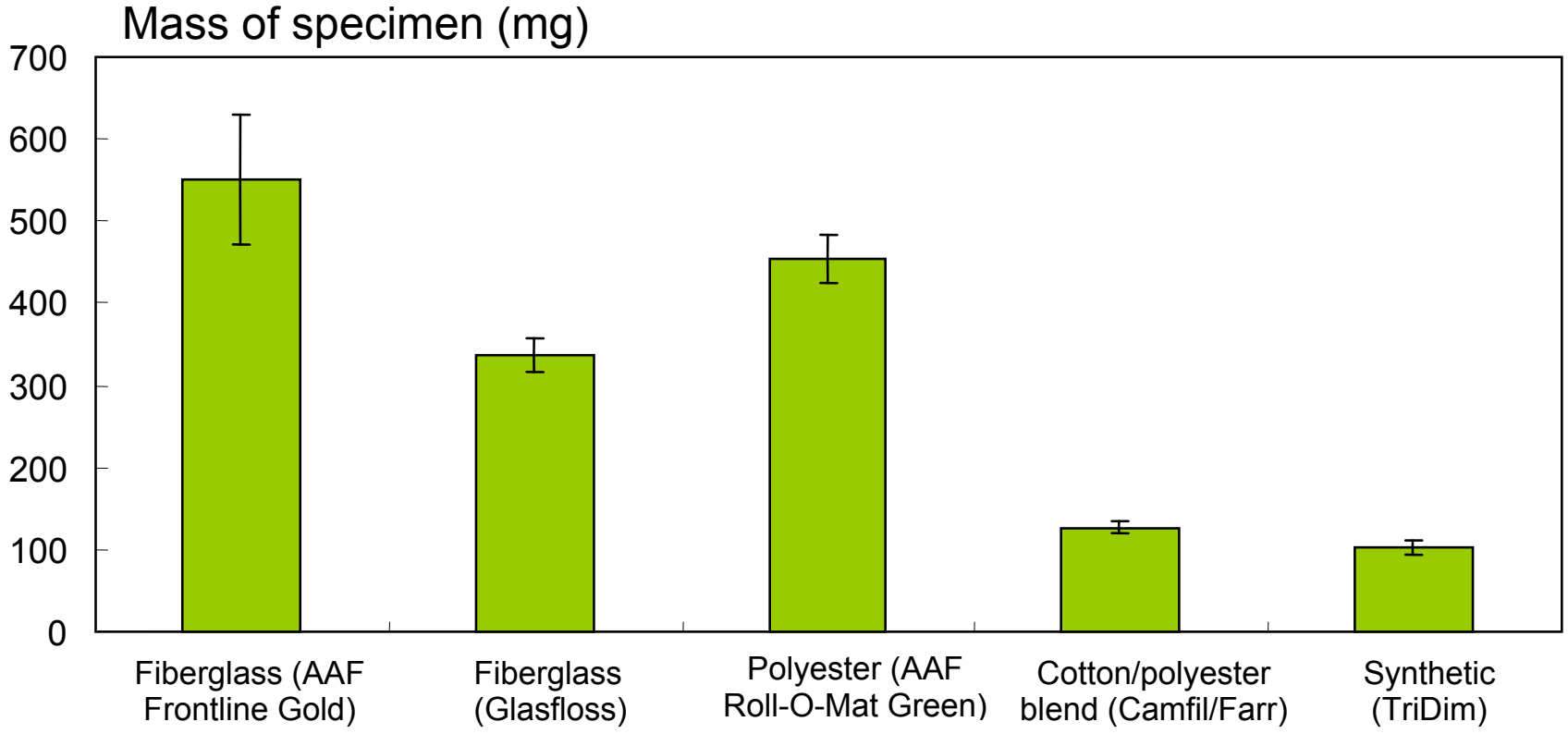

Figure 2: Mass of selected filter specimens used in this study (the error bars correspond to one standard deviation).

\section{B.2. Experimental setup}

The experimental setup designed and used in this study is shown in Figure 3. A clean airflow was split into two similar streams, one of which was run through a filter media sample, while the other was used as a reference. The ozone level was controlled with an ozone generator (UVP, Upland, California) upstream of the filters, and the humidity was adjusted by circulating part of the incoming airflow through a water bubbler (humidifier). Water-saturated air at the bubbler was diluted with a similar flow of dry air to avoid the entrainment of water droplets from the bubbler, obtaining $\mathrm{RH} \sim 50 \%$. The ozone level was measured at 5-min intervals, alternatively at the filter and the reference flow, using a photometric monitor (API 400). A multiplexing valve was used to switch from the filter to the reference. Gas phase samples were collected through ports located downstream 
of the exposed filters and on the reference line. The experimental setup was operated under room temperature, in the range $21-24{ }^{\circ} \mathrm{C}$, controlled by the laboratory thermostat. System temperature and $\mathrm{RH}$, as well as ozone concentrations, were recorded continuously in a data logging system (APT, The Energy Conservatory, Minneapolis, MN). Stainless steel, Teflon tubing, Teflonlined Tygon tubing and Teflon filter holders were used to minimize ozone loss to the surfaces of the flow system.

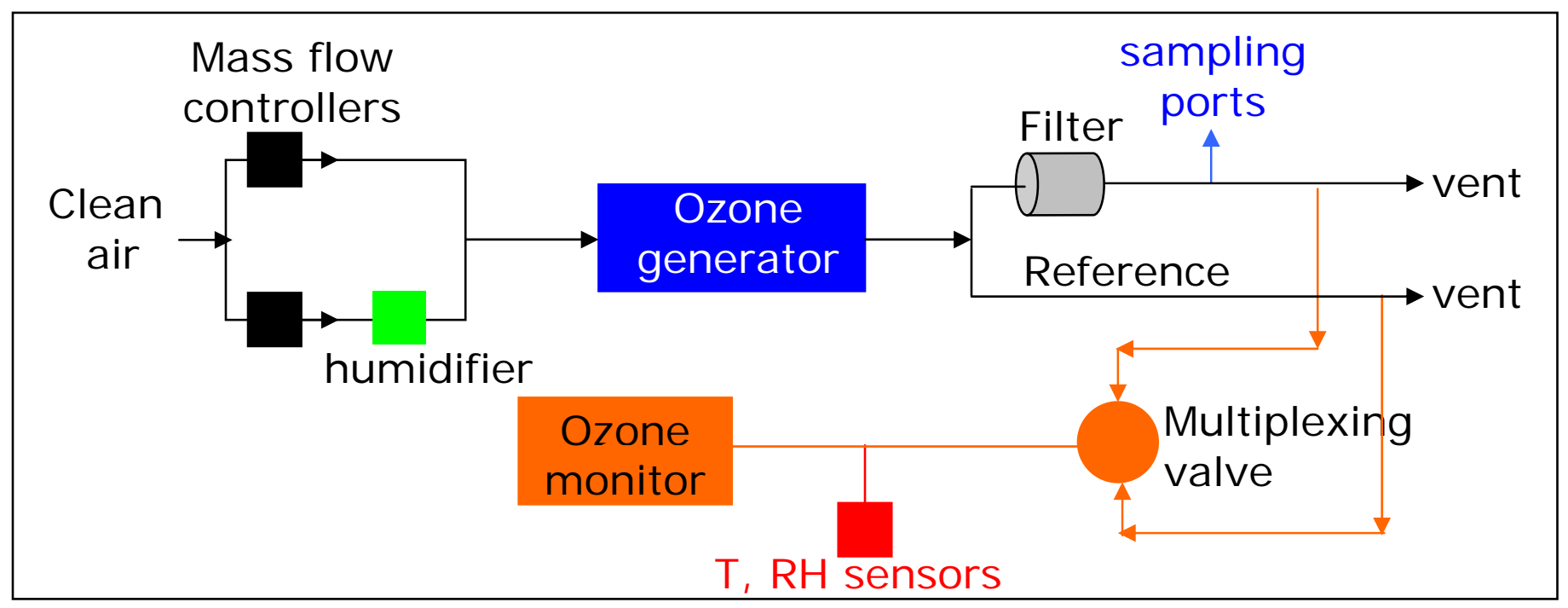

Figure 3: Experimental setup

Figure 4 illustrates the preparation of a typical specimen, and its placement in the Teflon holder and flow tube. 


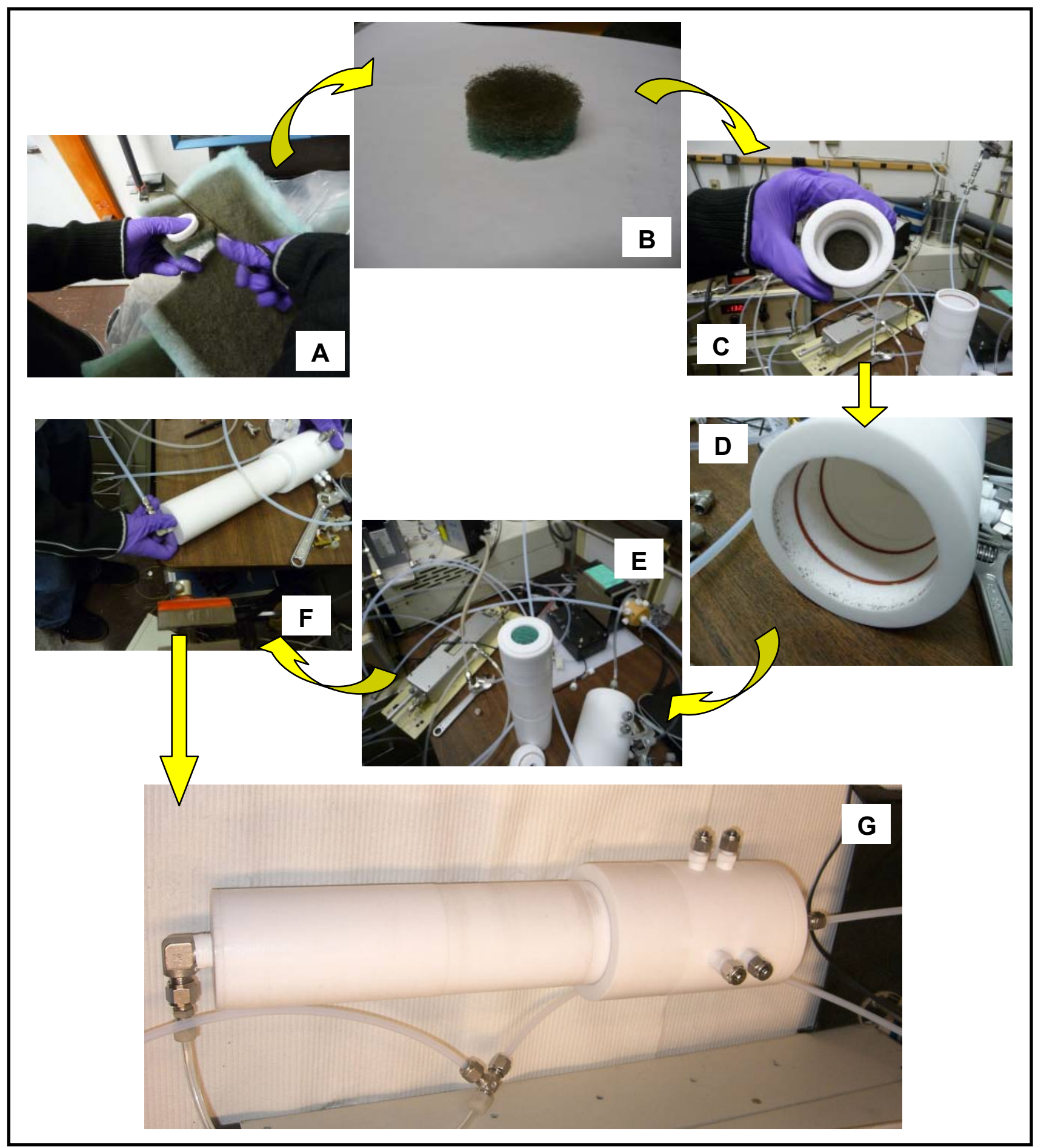

Figure 4: Preparation of sample. (A) a specimen is cut from a filter; $(B)$ the sample is weighed; (C) the sample is inserted in a Teflon holder; (D) the holder is inserted in the flow tube using Teflon-coated o-rings; (E,F): the flow tube is closed, and (G) the flow tube is connected to the experimental setup. 


\section{B.3. Ozone breakthrough curves}

Used and unused HVAC filters were exposed to air containing $150 \mathrm{ppbv}$ (with a precision of $\pm 5 \mathrm{ppbv}$ ) of ozone at the inlet for periods of $\sim 1000 \mathrm{~min}$. In each case, two experiments were performed, with dry and humidified air (at $50 \pm 5$ $\% \mathrm{RH}$ ). The airflow through the filter was $1.3 \mathrm{~L} / \mathrm{min}$, corresponding to a face velocity of $0.013 \mathrm{~m} / \mathrm{s}(2.56 \mathrm{ft} / \mathrm{min})$. We recorded ozone breakthrough curves by measuring $\left[\mathrm{O}_{3}\right]$ levels downstream of the filter and in the reference line (equivalent to upstream ozone levels), at 5-minute intervals. The difference in ozone concentration between each period corresponding to downstream of the filter and the average of the reference periods immediately before and after was recorded as $\Delta\left[\mathrm{O}_{3}\right]$.

We also performed a few experiments at a higher airflow rate, corresponding to a face velocity of $0.093 \mathrm{~m} / \mathrm{s}$ (or $18.3 \mathrm{ft} / \mathrm{min}$ ), to compare our results with conditions that are slightly closer to HVAC usual operation conditions. However, even this higher flow is roughly one order of magnitude lower than typical HVAC conditions.

\section{B.4. Determination of formaldehyde and acetaldehyde emissions}

In samples placed downstream of the filters, we collected volatile carbonyls formed in the oxidation of filter media and dust particles deposited on the filters. The integrated aldehyde measurements were performed across the entire time frame of each ozone-filter test, including both the initial and steady-state periods. The samplers (Waters model \# WAT047205) consisted of silica gel cartridges impregnated with DNPH (dinitro phenylhydrazine), a derivatization reactant specific to carbonyls. DNPH samplers were preceded by an ozone scrubber (Waters model \# WAT054420) to remove ozone from the sampling stream, following protocols that have been previously tested in our laboratory. The flow through the sampler was measured immediately before starting and at the end of the sampling period, and was used, together with the sampling time, to determine the volume of air that was sampled. Flow through DNPH samplers were constant during sampling, with fluctuations that were typically below $3 \%$. The exposed samplers were extracted with 2-mL acetonitrile and analyzed by HPLC with UV detection (Agilent 1200 system). Formaldehyde and acetaldehyde were quantified with a calibration curve prepared with authentic standards of the DNPH hydrazone derivatives. 


\section{C. $\underline{\text { Results }}$}

\section{C.1. Ozone breakthrough curves}

Figure 5 illustrates a typical ozone breakthrough experiment with humidified air, performed on a Glasfloss synthetic filter used in the Port of Oakland. The curve in red corresponds to ozone concentration in the reference airflow, and the curve in blue to the ozone measured downstream of the sample. Each data point is the average of a 5-min measurement. The change in ozone concentration $\left(\Delta\left[\mathrm{O}_{3}\right]\right)$ can be calculated from the difference between those two curves. The green curve represents the percent change in ozone, $\% \Delta\left[\mathrm{O}_{3}\right]$,

$$
\% \Delta\left[\mathrm{O}_{3}\right]=\frac{\Delta\left[\mathrm{O}_{3}\right]}{\left[\mathrm{O}_{3}\right]_{\text {ref }}} \times 100
$$

with respect to inlet (i.e., reference) ozone concentration, $\left[\mathrm{O}_{3}\right]_{\text {ref. }}$ Our experimental approach allowed us to obtain very precise values of $\Delta\left[\mathrm{O}_{3}\right]$ over long periods of time, even in the presence of fluctuations due to flow or lamp intensity instabilities.

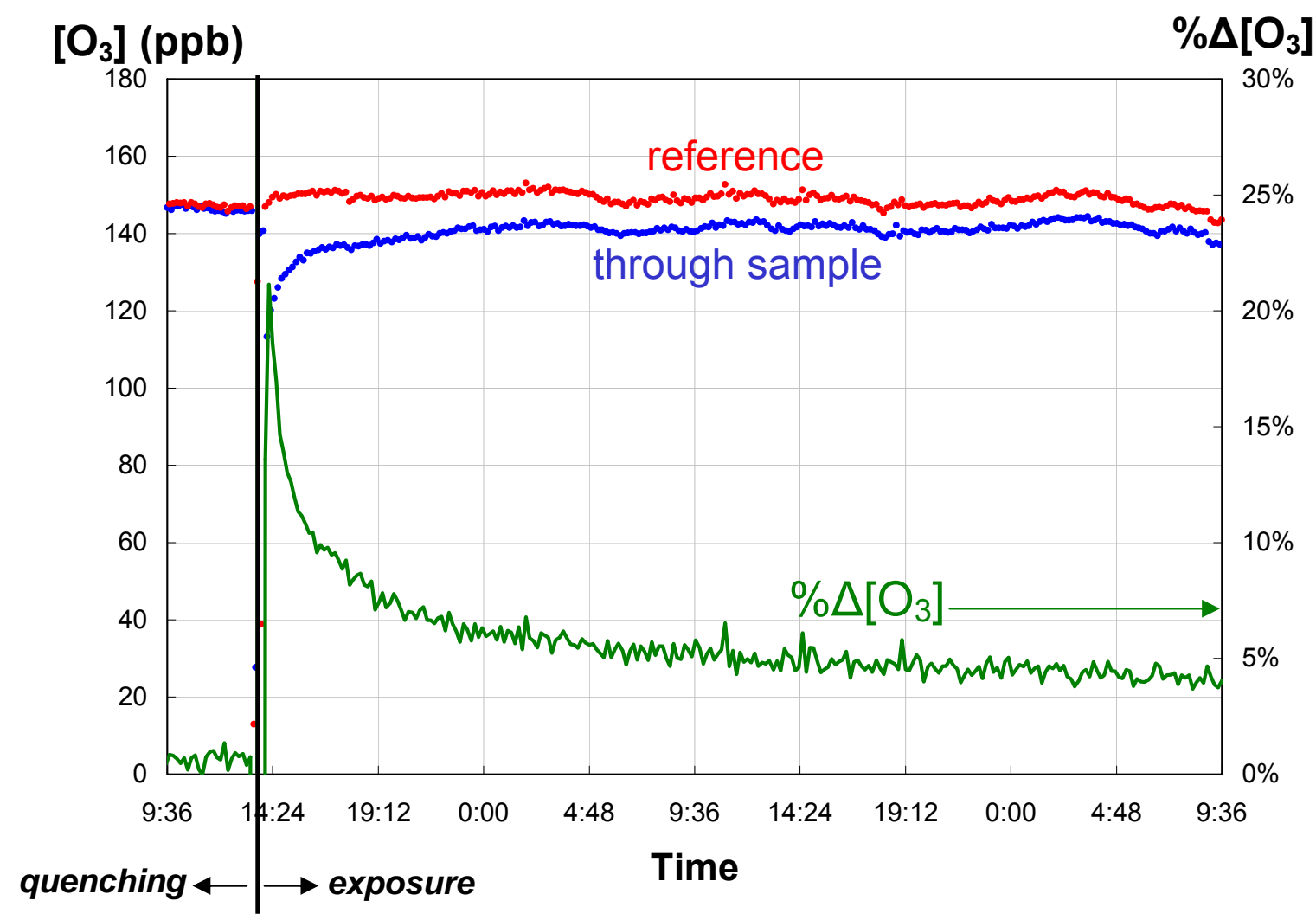

Figure 5: Example of ozone breakthrough curves obtained in a typical experiment. These results correspond to a Glasfloss synthetic filter used in the Port of Oakland, exposed to $150 \mathrm{ppb}$ ozone under humidified air. 
Results from ozone breakthrough curves were adjusted to the exponential fitting:

$$
\Delta\left[\mathrm{O}_{3}\right]=A \cdot \exp (-B t)+\Delta\left[\mathrm{O}_{3}\right]_{\text {st.state }}
$$

which allowed for determination of the time at which outlet ozone concentration approached steady state $\left(t_{\text {st.state. }}\right)$, the average ozone concentration change $\Delta\left[\mathrm{O}_{3}\right]_{\text {initial }}$ with respect to inlet concentrations, corresponding to the initial period (i.e., for $\mathrm{t}<t_{\text {st.st. }}$ ), and $\Delta\left[\mathrm{O}_{3}\right]_{\text {st.state }}$, the ozone concentration change corresponding to the steady state period (i.e., for $t>t_{\text {st.st. }}$ ). The value of $t_{\text {st.st. }}$ in each experiment was established as the time at which the fitted $\Delta\left[\mathrm{O}_{3}\right]$ was $5 \%$ higher than the fitted $\Delta\left[\mathrm{O}_{3}\right]_{\text {st.state. }}$ These parameters, obtained from each experiment, are illustrated generically in Figure 6.

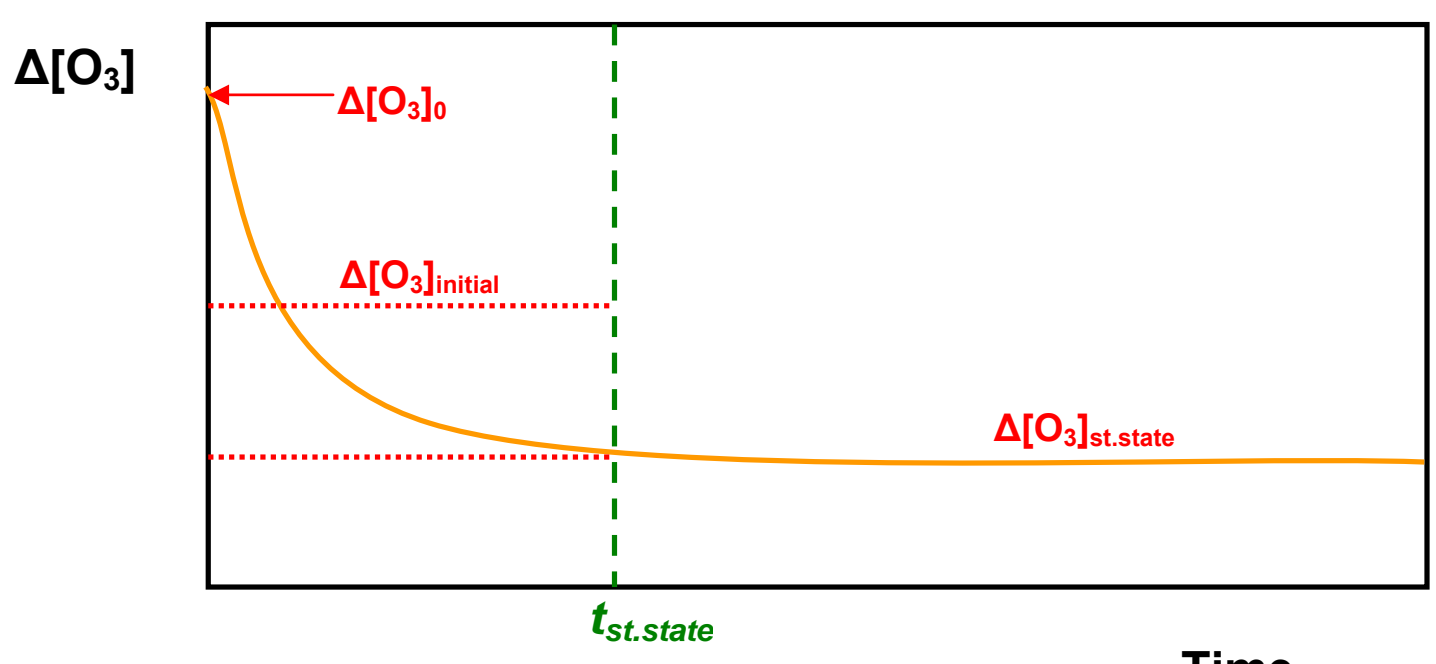

Figure 6: Schematic representation of an ozone breakthrough curve, showing the parameters obtained from fitting the experimental data.

The experimental conditions, the $t_{\text {st.state }}$ values and the average ozone concentration drops $\Delta\left[\mathrm{O}_{3}\right]$ are reported in Table 2 (for the unused filters) and in Table 3 (for the used filters). Ozone concentrations are calculated as averages over each of the periods considered in the experiments. Tables 2 and 3 also include the mass of ozone consumed during the initial period (in $\mu \mathrm{g} \mathrm{O}_{3}$ ), and the rate of ozone consumption (in $\mu \mathrm{g} \mathrm{O}_{3} \mathrm{~h}^{-1}$ ) at steady state conditions. 
Table 2: Experimental results obtained from ozone breakthrough curves for unused filters

\begin{tabular}{|c|c|c|c|c|c|c|c|}
\hline \multirow{3}{*}{$\begin{array}{c}\text { Manufacturer/ } \\
\text { Model }\end{array}$} & \multirow{2}{*}{ Inlet ozone } & \multirow{2}{*}{$\mathbf{R H}$} & \multirow{2}{*}{$t_{\text {st.state }}$} & \multicolumn{2}{|c|}{$\Delta\left[\mathrm{O}_{3}\right]$} & \multicolumn{2}{|c|}{ Mass of $0_{3}$ reacted } \\
\hline & & & & Initial & St.State & Initial & St.State \\
\hline & (ppb) & $(\%)$ & $(\min )$ & (ppb) & (ppb) & $(\mu \mathrm{g})$ & $\left(\mu \mathrm{g} \mathrm{h}^{-1}\right)$ \\
\hline \multicolumn{8}{|c|}{ Fiberglass } \\
\hline AAF Frontline Green ${ }^{(a)}$ & $146 \pm 9$ & 0 & 552 & 13.6 & 5.81 & 8.26 & 0.90 \\
\hline AAF Frontline Green ${ }^{(a)}$ & $151 \pm 6$ & 53 & 106 & 13.1 & 4.54 & 3.11 & 0.70 \\
\hline AAF Frontline Gold ${ }^{(a)}$ & $161 \pm 9$ & 0 & 529 & 11.9 & 3.43 & 3.24 & 0.53 \\
\hline AAF Frontline Gold ${ }^{(a)}$ & $147 \pm 7$ & 52 & 422 & 13.3 & 3.61 & 3.02 & 0.56 \\
\hline AAF Frontline Blue & $137 \pm 16$ & 0 & 237 & 4.88 & n.d. & 2.16 & n.d. \\
\hline AAF Frontline Blue & $144 \pm 6$ & 52 & 193 & 6.37 & n.d. & 1.94 & n.d. \\
\hline Glasfloss Disposable & $145 \pm 6$ & 0 & 209 & 1.93 & n.d. & 2.80 & n.d. \\
\hline Glasfloss Disposable & $144 \pm 5$ & 52 & 72 & 4.65 & n.d. & 1.21 & n.d. \\
\hline Flanders EZ Flow II & $180 \pm 3$ & 0 & 160 & 2.85 & n.d. & 1.17 & n.d. \\
\hline Flanders EZ Flow II & $162 \pm 1$ & 68 & 122 & 7.50 & n.d. & 2.41 & n.d. \\
\hline \multicolumn{8}{|c|}{ Polyester } \\
\hline AAF Roll-o-Mat green ${ }^{(b)}$ & $148 \pm 3$ & 0 & 228 & 4.36 & 0.49 & 2.98 & 0.08 \\
\hline AAF Roll-o-Mat green ${ }^{(b)}$ & $154 \pm 7$ & 59 & 263 & 2.71 & n.d. & 1.58 & n.d. \\
\hline AAF Roll-o-Mat green ${ }^{(c)}$ & $165 \pm 3$ & 0 & 59 & 5.57 & n.d. & 2.11 & n.d. \\
\hline AAF Roll-o-Mat green ${ }^{(c)}$ & $155 \pm 3$ & 57 & 145 & 4.54 & n.d. & 0.72 & n.d. \\
\hline \multicolumn{8}{|c|}{ Cotton/polyester blend } \\
\hline Airguard DP 40 & $153 \pm 13$ & 0 & 168 & 3.06 & n.d. & 4.16 & n.d. \\
\hline Airguard DP 40 & $145 \pm 5$ & 54 & 93 & 7.35 & n.d. & 2.24 & n.d. \\
\hline Camfil Farr 30/50 & $141 \pm 16$ & 0 & 164 & 9.48 & 1.24 & 4.81 & 0.20 \\
\hline Camfil Farr 30/50 & $145 \pm 4$ & 54 & 119 & 7.10 & 1.29 & 1.33 & 0.20 \\
\hline \multicolumn{8}{|c|}{ Synthetic } \\
\hline Tri-Dim ES-40 & $136 \pm 6$ & 0 & 100 & 4.45 & 1.25 & 4.97 & 0.20 \\
\hline Tri-Dim ES-40 & $145 \pm 9$ & 52 & 88 & 4.78 & 1.10 & 2.59 & 0.17 \\
\hline Glasfloss Z line ZL & $141 \pm 7$ & 0 & 91 & 3.34 & n.d. & 0.79 & n.d. \\
\hline Glasfloss Z line ZL & $145 \pm 4$ & 53 & 61 & 2.64 & 0.64 & 0.43 & 0.10 \\
\hline
\end{tabular}

n.d.: $\Delta\left[\mathrm{O}_{3}\right]$ not detected; ozone concentration downstream of the filter was not significantly different from the reference.

(a) these filters were tested more than once

(b) without plastic backing

(c) with plastic backing 
In Figure 7 we present the average ozone concentration drop $\Delta\left[\mathrm{O}_{3}\right]$ reported in Table 2 for the unused filters, by filter category, during the initial and steady-state periods. In all cases, initial ozone reactivity was higher than under steady-state conditions, illustrating that the most reactive constituents present on the surface of the filter media were rapidly eliminated by reaction with ozone.

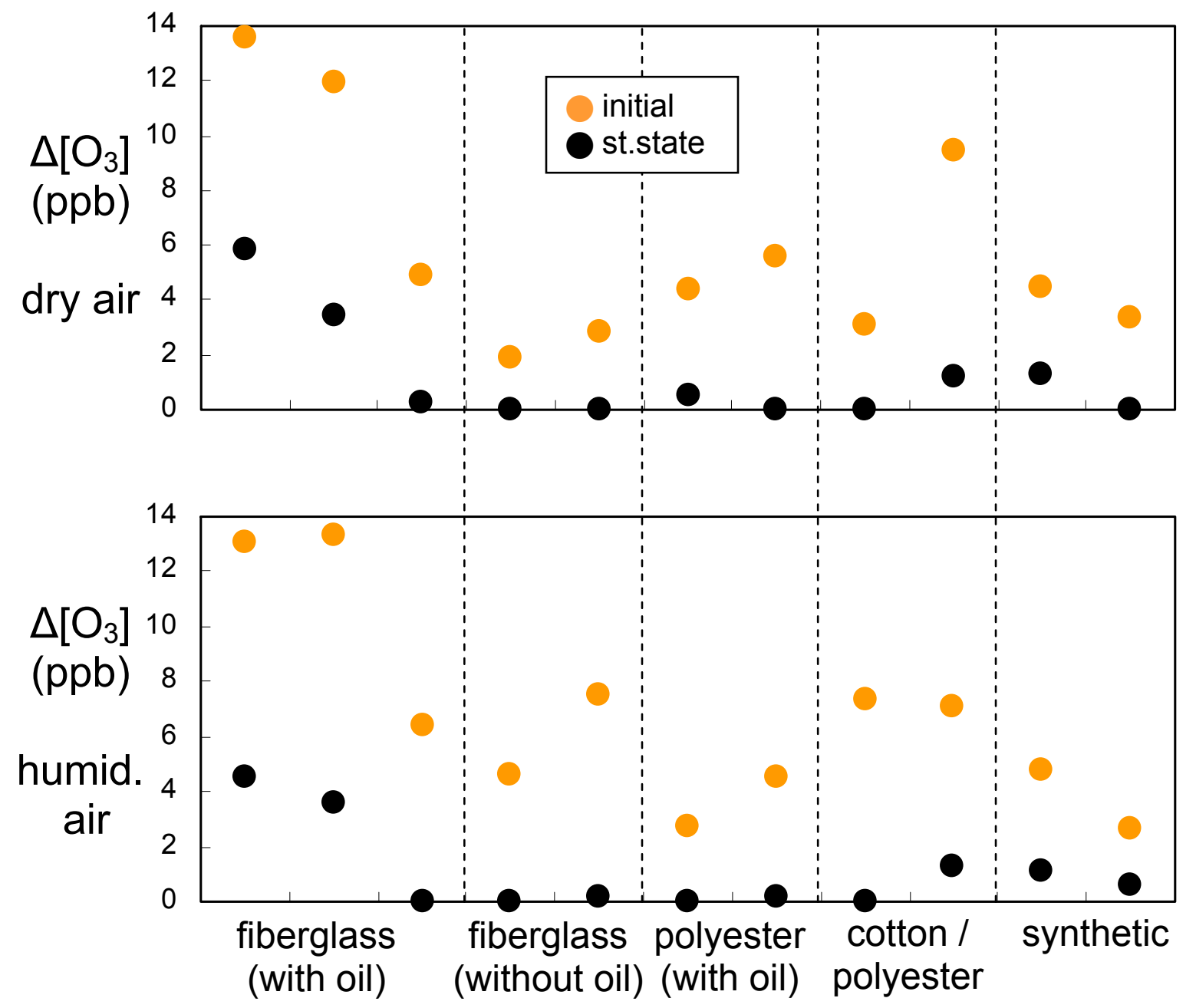

Figure 7: Initial and steady-state ozone reaction reduction $\Delta\left[\mathrm{O}_{3}\right]$ in unused filters. 
Table 3: Experimental results obtained from ozone breakthrough curves for used filters

\begin{tabular}{|c|c|c|c|c|c|c|c|c|}
\hline \multirow{3}{*}{$\begin{array}{l}\text { Manufacturerl } \\
\text { Model }\end{array}$} & \multirow{3}{*}{ Building } & \multirow{2}{*}{$\begin{array}{l}\text { Inlet } \\
\text { ozone }\end{array}$} & \multirow{2}{*}{$\mathbf{R H}$} & \multirow{2}{*}{$t_{\text {st.state }}$} & \multicolumn{2}{|c|}{$\Delta\left[\mathrm{O}_{3}\right]$} & \multicolumn{2}{|c|}{ Mass of $0_{3}$ reacted } \\
\hline & & & & & Initial & St.State & Initial & St.State \\
\hline & & (ppb) & (\%) & $(\min )$ & (ppb) & (ppb) & $(\mu g)$ & $\left(\mu \mathrm{g} \mathrm{h}^{-1}\right)$ \\
\hline \multicolumn{9}{|c|}{ Fiberglass } \\
\hline AAF Frontline Gold & LBNL90 & $161 \pm 5$ & 0 & 422 & 40.2 & 1.46 & 39.9 & 0.21 \\
\hline AAF Frontline Gold & LBNL90 & $171 \pm 4$ & 64 & 849 & 18.1 & 5.79 & 39.2 & 0.89 \\
\hline AAF Frontline Gold & Port & $167 \pm 3$ & 0 & 1276 & 26.1 & 10.1 & 84.7 & 1.54 \\
\hline AAF Frontline Gold & Port & $176 \pm 12$ & 55 & 1182 & 42.6 & 23.9 & 129.1 & 3.67 \\
\hline Glasfloss Disposable & LBNL46 & $162 \pm 5$ & 0 & 158 & 20.5 & n.d. & 8.28 & n.d. \\
\hline Glasfloss Disposable & LBNL46 & $150 \pm 2$ & 59 & 289 & 7.25 & 1.42 & 5.39 & 0.22 \\
\hline Glasfloss Disposable & Port & $173 \pm 2$ & 0 & 189 & 6.37 & 0.32 & 1.04 & 0.05 \\
\hline Glasfloss Disposable & Port & $146 \pm 5$ & 57 & 327 & 10.2 & 5.02 & 18.7 & 0.73 \\
\hline \multicolumn{9}{|c|}{ Polyester } \\
\hline$\underset{\text { (a) }}{\text { AAF Roll-o-Mat green }}$ & LBNL90 & $171 \pm 7$ & 0 & 322 & 23.4 & 8.23 & 19.6 & 1.28 \\
\hline$\underset{\text { (a) }}{\text { AAF Roll-o-Mat green }}$ & LBNL90 & $160 \pm 2$ & 62 & 315 & 16.9 & 10.2 & 30.5 & 1.42 \\
\hline AAF Roll-o-Mat green & Port & $150 \pm 2$ & 0 & 502 & 15.8 & 4.20 & 20.5 & 0.65 \\
\hline$\underset{\text { (a) }}{\text { AAF Roll-o-Mat green }}$ & Port & $144 \pm 2$ & 49 & 1146 & 15.3 & 9.60 & 45.7 & 1.51 \\
\hline \multicolumn{9}{|c|}{ Cotton/polyester blend } \\
\hline Airguard DP 40 & LBNL90 & $160 \pm 4$ & 0 & 192 & 3.58 & 0.43 & 1.62 & 0.06 \\
\hline Airguard DP 40 & LBNL90 & $197 \pm 1$ & 55 & 64 & 26.64 & 0.97 & 4.40 & 0.15 \\
\hline Camfil Farr 30/50 & LBNL90 & $149 \pm 13$ & 0 & 104 & 3.34 & 0.18 & 0.81 & 0.02 \\
\hline Camfil Farr 30/50 & LBNL90 & $149 \pm 2$ & 60 & 93 & 5.18 & 0.48 & 1.24 & 0.07 \\
\hline Camfil Farr 30/50 & Port & $168 \pm 2$ & 0 & 261 & 10.55 & 3.77 & 7.14 & 0.59 \\
\hline Camfil Farr 30/50 & Port & $152 \pm 2$ & 47 & 492 & 8.15 & 4.03 & 10.33 & 0.62 \\
\hline \multicolumn{9}{|c|}{ Synthetic } \\
\hline Tri-Dim ES-40 & LBNL90 & $152 \pm 2$ & 0 & 201 & 4.55 & n.d. & 2.39 & n.d. \\
\hline Tri-Dim ES-40 & LBNL90 & $140 \pm 2$ & 51 & 175 & 4.51 & 0.38 & 2.09 & 0.06 \\
\hline Glasfloss Z line ZL & Port & $161 \pm 5$ & 0 & 272 & 21.76 & 2.76 & 15.53 & 0.44 \\
\hline Glasfloss Z line ZL & Port & $149 \pm 2$ & 54 & 501 & 12.98 & 3.66 & 17.06 & 0.58 \\
\hline
\end{tabular}

n.d.: $\Delta\left[\mathrm{O}_{3}\right]$ not detected; ozone concentration downstream of the filter was not significantly different from the reference (a) with plastic backing

The information presented in Tables 2 and 3 serves as a basis for a preliminary evaluation of the ozone removal capacity of HVAC filters. Evaluation of our 
results should be mindful that, in buildings, ozone exposure and pollutant loading on the filters may occur gradually and simultaneously over time, rather than in the sequential pattern used in our experiments. In all cases we observed an initially higher ozone loss rate, which is subsequently reduced to a lower but, in most cases, non-zero value. The times required to reach steady state conditions with unused filters were in the range 59-552 minutes. For used filters longer times were often observed, particularly for fiberglass and polyester filters at humidified conditions (>1000 minutes in a few cases). Some new fiberglass media showed high reactivity with ozone, but all four new filter categories showed relatively similar trends. Used filters presented significantly higher ozone uptake than unused filters of the same type, particularly when exposed to humidified air. Samples from the Port of Oakland were loaded with carbonaceous materials (imparting a characteristic darker color), and led to significantly higher ozone uptake, as compared with similar filters deployed in LBNL buildings. A comparison of ozone uptake during the initial 24-h ozone exposure period for each filter type, unused or used at LBNL or the Port of Oakland, is shown in Figures $8-10$. In these figures, the mass of ozone uptake during the initial period was obtained directly from Table 3 . The uptake of ozone between $t_{\text {st.state }}$ and $t=24 \mathrm{~h}$ was calculated using the rate of ozone uptake reported in Table 3 for each filter.

Highest ozone uptake rates were observed for fiberglass filters, particularly for AAF Frontline media. Although these were the filters with highest mass per unit surface (as shown in Figure 2), the mass difference does not account for the much higher ozone uptake observed in these filters. The presence of a coating of Viscosine impaction oil (ranging from a light coating on the AAF Frontline Green media to a heavy coating on the AAF Frontline Gold media) is likely a reason for the higher ozone uptake. The AAF Roll-O-Mat Green polyester filter media, treated with a medium application of Viscosine impaction oil, also exhibited also a high ozone uptake rate both under humidified and dry air conditions, with a significant contribution of the steady-state period, which indicates that this media can be a moderate but constant source of indoor pollutants originated in ozone chemistry. In Figures 9 and 10, results from cotton/polyester and synthetic media suggest that those filters may be a less significant source of secondary pollutants from ozone reactions. Only the samples used in the Port of Oakland showed significant ozone uptake, likely related to the carbonaceous nature of the material collected on the filter surfaces. 
$\mu \mathrm{g} \mathrm{O}_{3}$ reacted

dry air
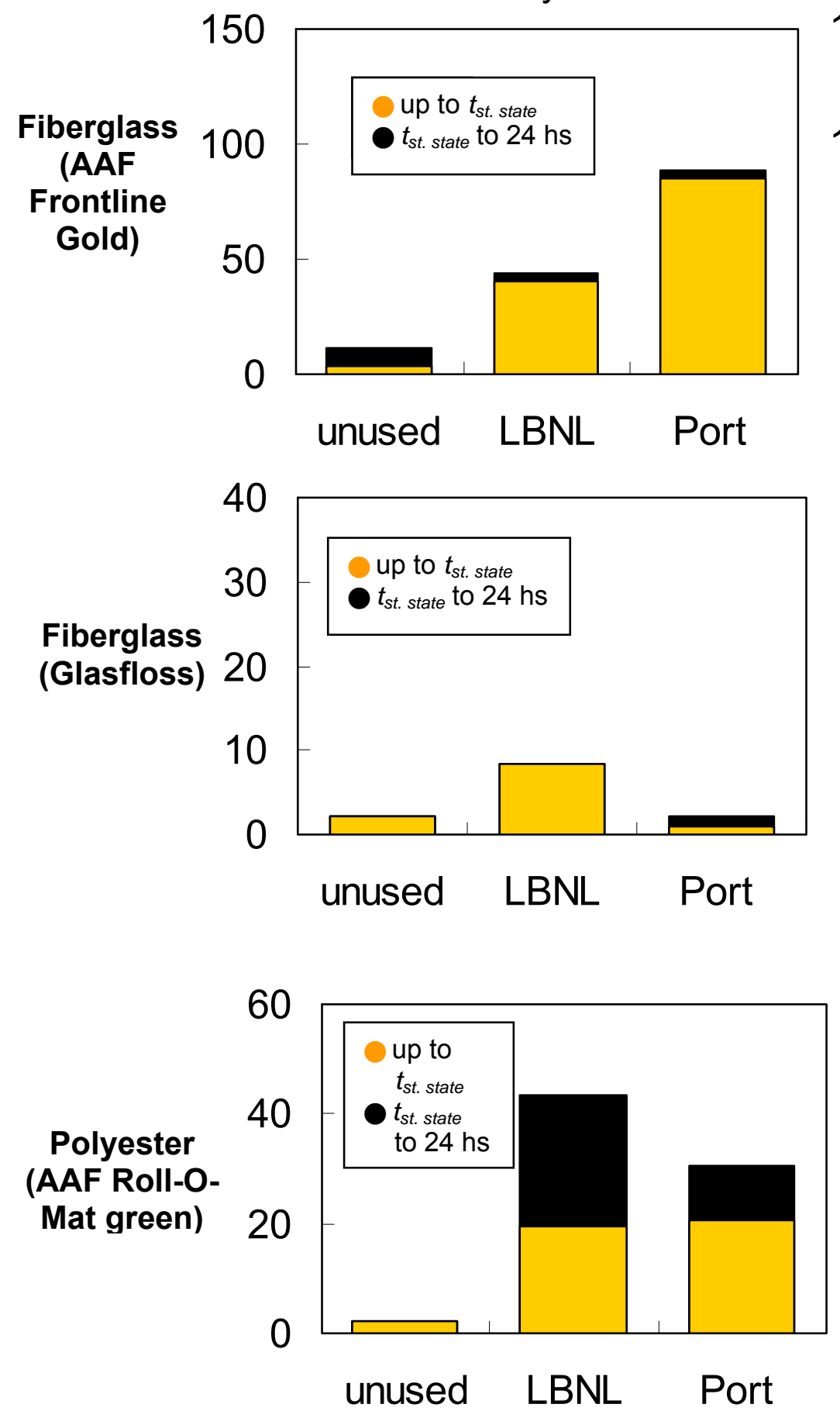

$\mu \mathrm{g} \mathrm{O}_{3}$ reacted

humidified air
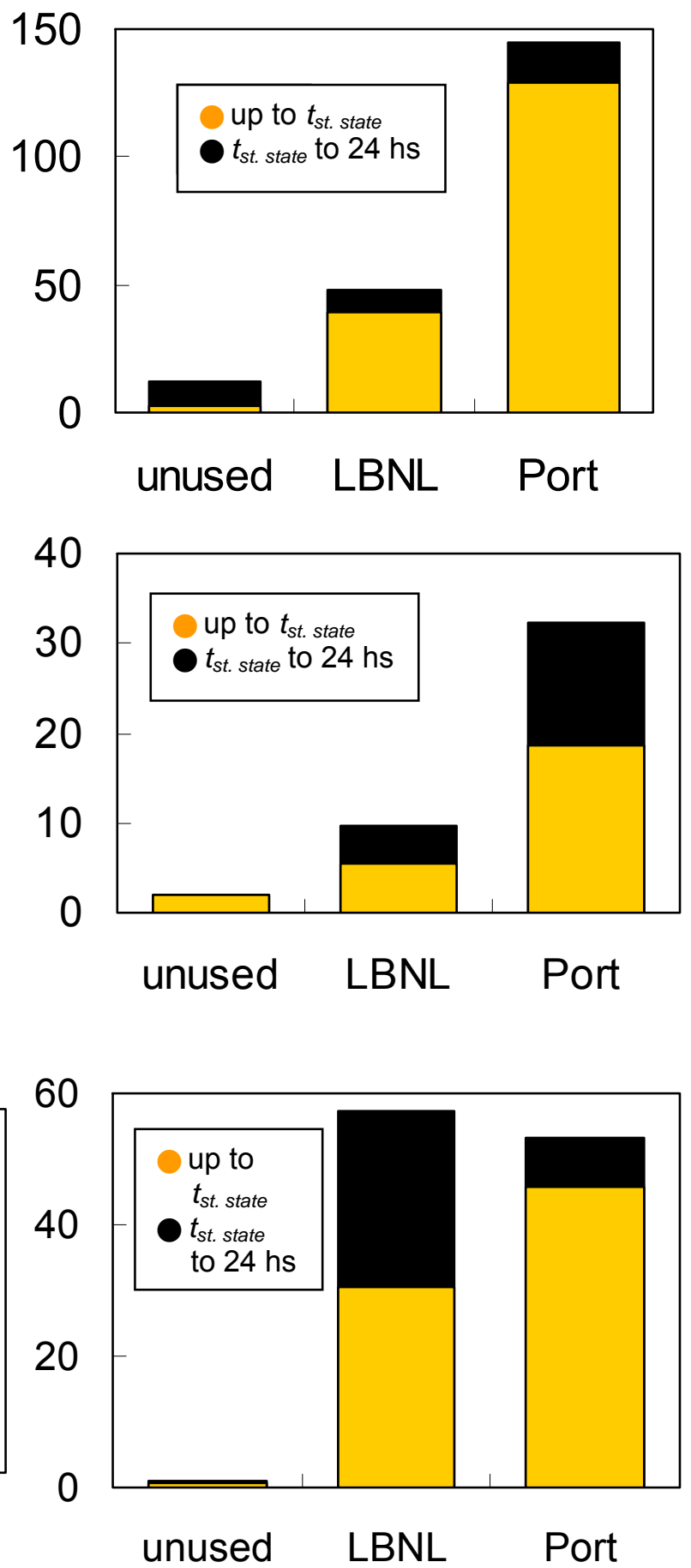

Figure 8: Ozone uptake in a 24-h period for two fiberglass filters and one polyester filter. 

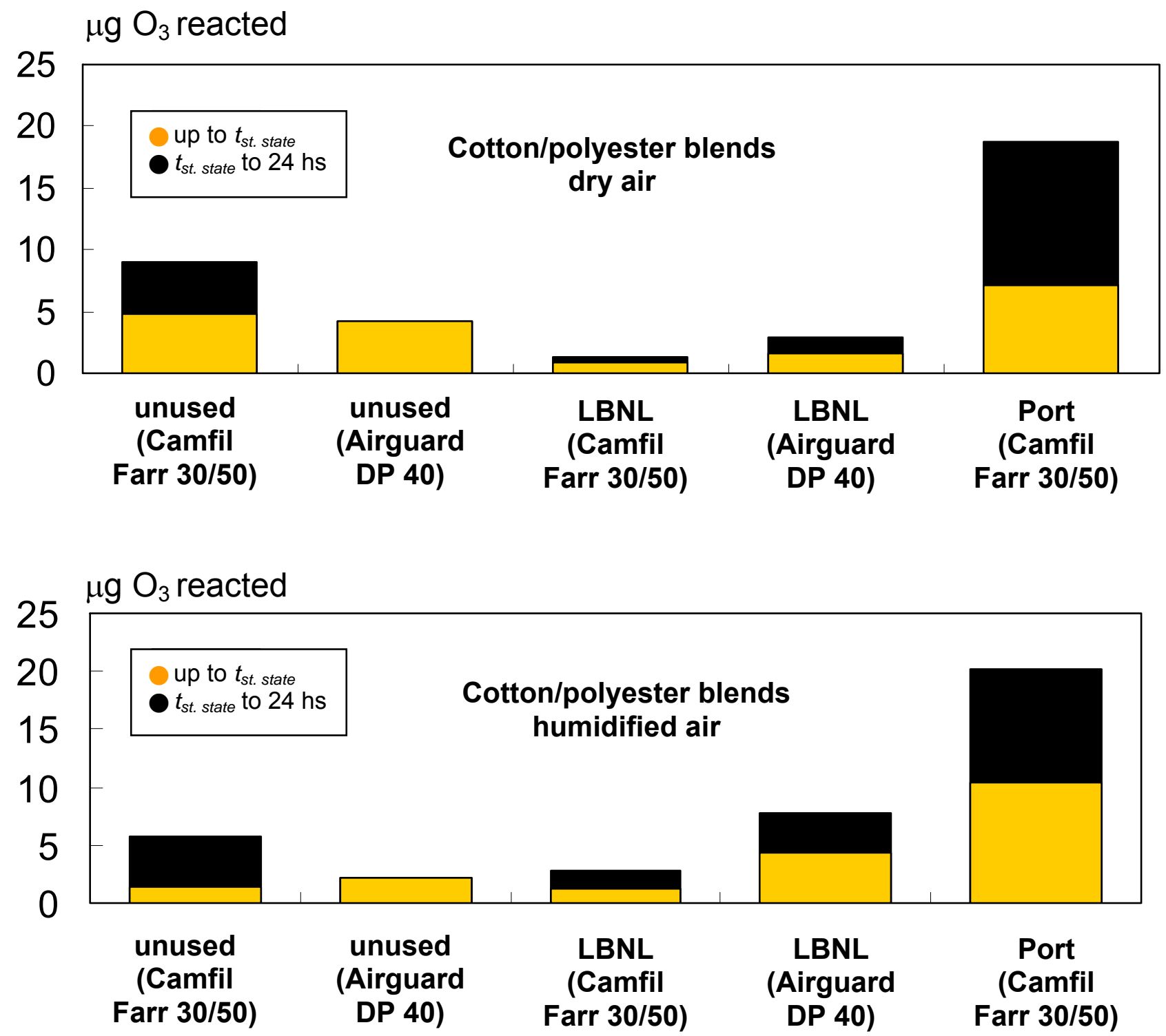

Figure 9: Ozone uptake in a 24-h period for cotton/polyester blend filters. 

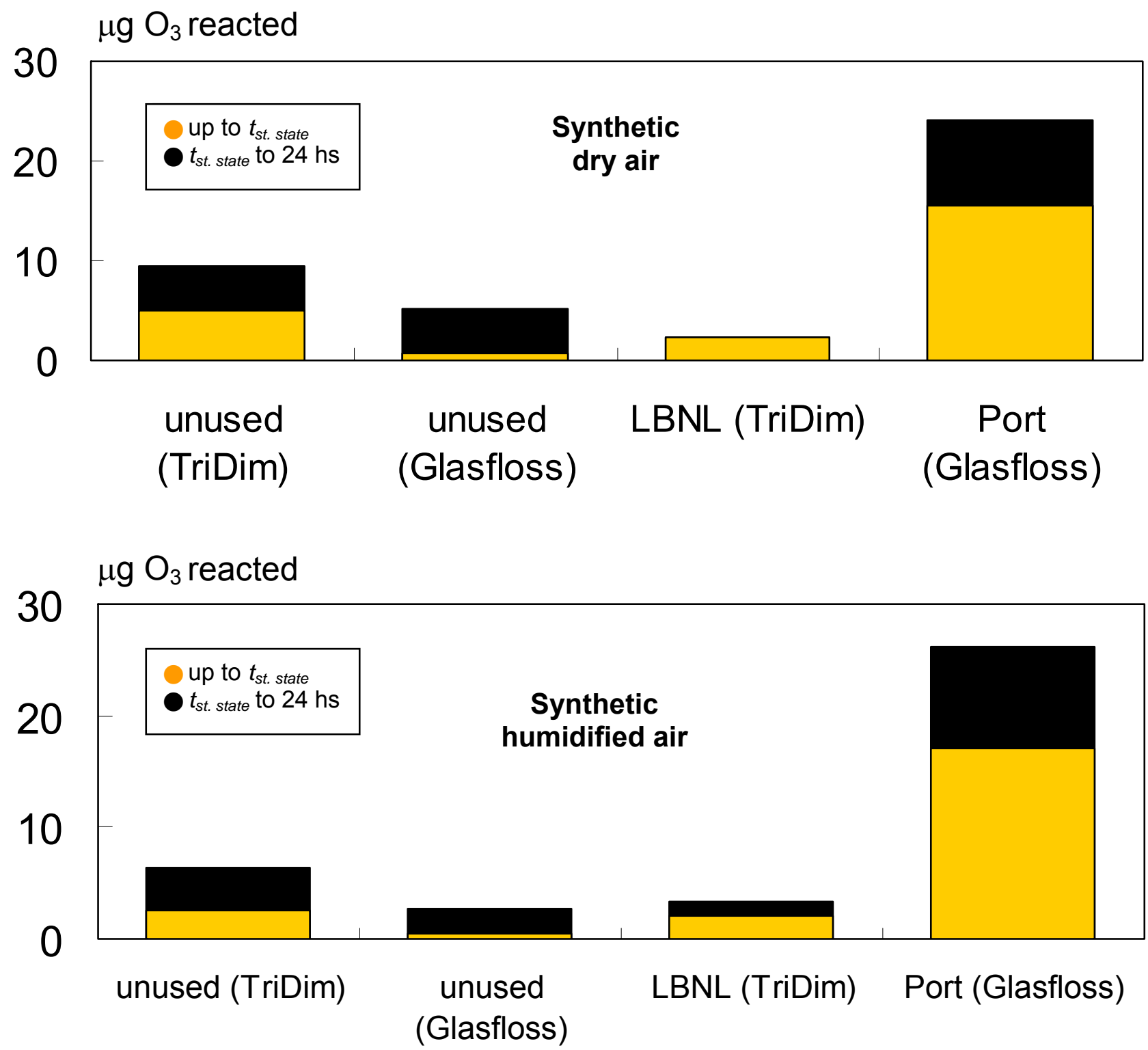

Figure 10: Ozone uptake in a 24-h period for synthetic filters. 


\section{C.2. Determination of formaldehyde and acetaldehyde emissions}

Results from initial DNPH experiments performed to evaluate the limit of detection of this method are shown in Figures 11 and 12. In these tests, we determined the limit of detection (laboratory blank) by measuring formaldehyde and acetaldehyde concentrations in the system operating without filters, under identical conditions as those used in the ozone breakthrough tests (i.e., with humidified air or dry air, and with $150 \mathrm{ppb}$ ozone or without ozone). In addition, we determined the reactants blank by extracting five DNPH cartridges that were not used in experiments.

[Formaldehyde]

$\left(\mu \mathrm{g} \cdot \mathrm{m}^{-3}\right)$

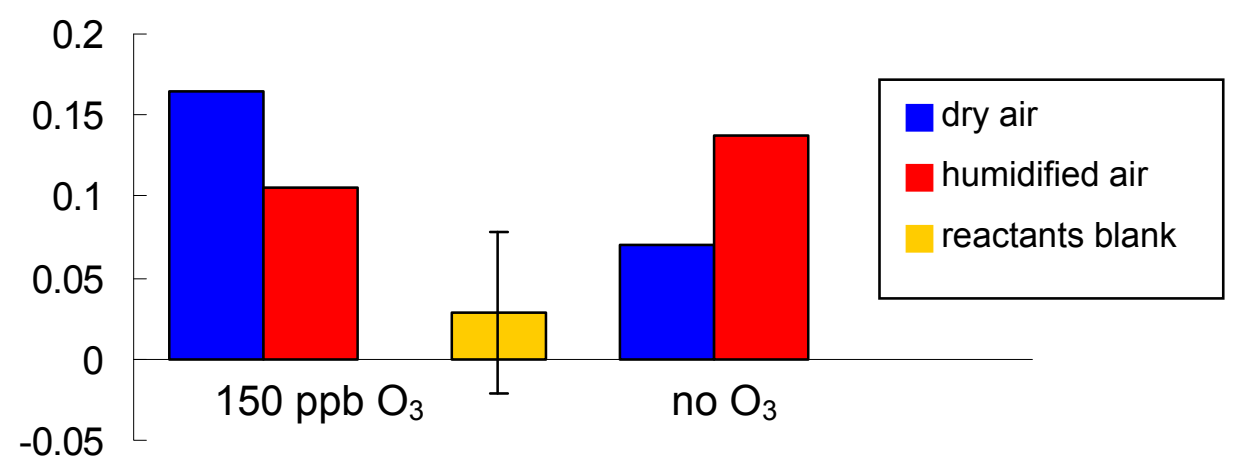

Figure 11: Tests performed without filters (laboratory blank), to evaluate the limit of detection of formaldehyde.

[Acetaldehyde]

$\left(\mu \mathrm{g} \cdot \mathrm{m}^{-3}\right)$

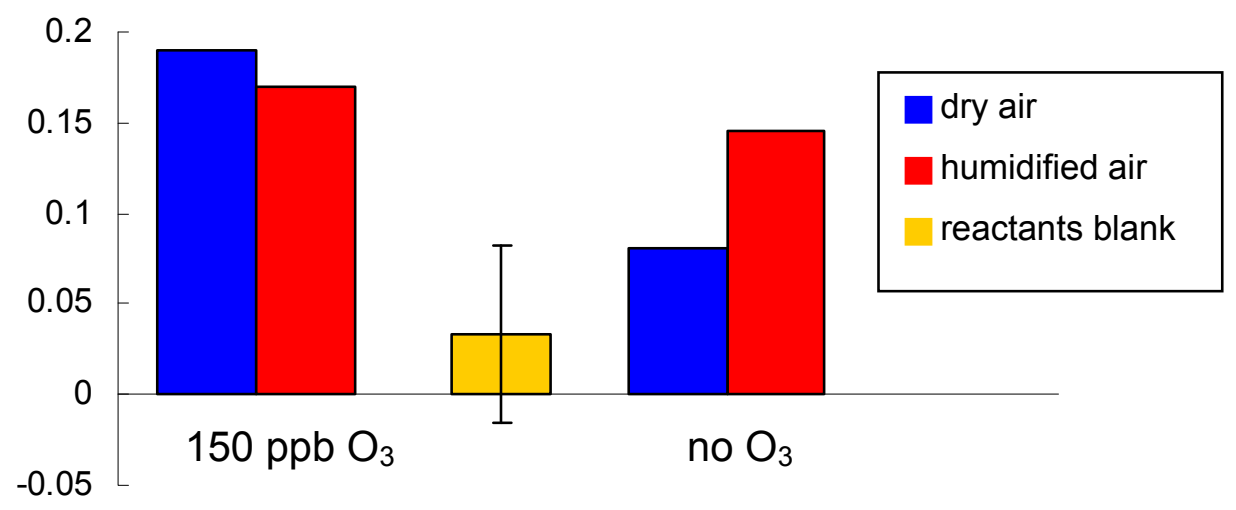

Figure 12: Tests performed without filters (laboratory blank), to evaluate the limit of detection of acetaldehyde. 
The average concentration of formaldehyde of the reactants blank was determined to be $0.02 \pm 0.05 \mu \mathrm{g} / \mathrm{m}^{3}$; the acetaldehyde concentration in the reactants blank was $0.04 \pm 0.05 \mu \mathrm{g} / \mathrm{m}^{3}$. In both cases, these tests also allowed us to evaluate the experimental uncertainty of this method, determined as one standard deviation. Figures 11 and 12 also show that formaldehyde and acetaldehyde concentrations in the laboratory blanks, determined by operating the experiment in the absence of HVAC filter media, were between 3 and 8 times higher than the reactants blank determinations. This difference is probably due uptake of aldehydes present in the laboratory air when the DNPH cartridges are handled during sampling and subsequent extraction.

Results obtained for experiments performed with unused filter media are presented in Figure 13 for formaldehyde and in Figure 14 for acetaldehyde. The inlet ozone concentrations ranged from 140 to $165 \mathrm{ppb}$. Overall, downstream aldehyde concentrations were low. For experiments performed using dry air, in nearly all cases formaldehyde and acetaldehyde concentrations were close or below the limit of detection, except for the results for the AAF Frontline Green filter, in which emissions were $\sim$ double the LOD for formaldehyde, and more than double the LOD for acetaldehyde. Experiments in humidified air showed consistently higher outlet aldehyde concentrations, ranging from $\sim 2$ to $\sim 7$ times higher than the limit of detection (which is indicated in each plot as a red dotted line (for humid air) and a blue dotted line (for dry air).

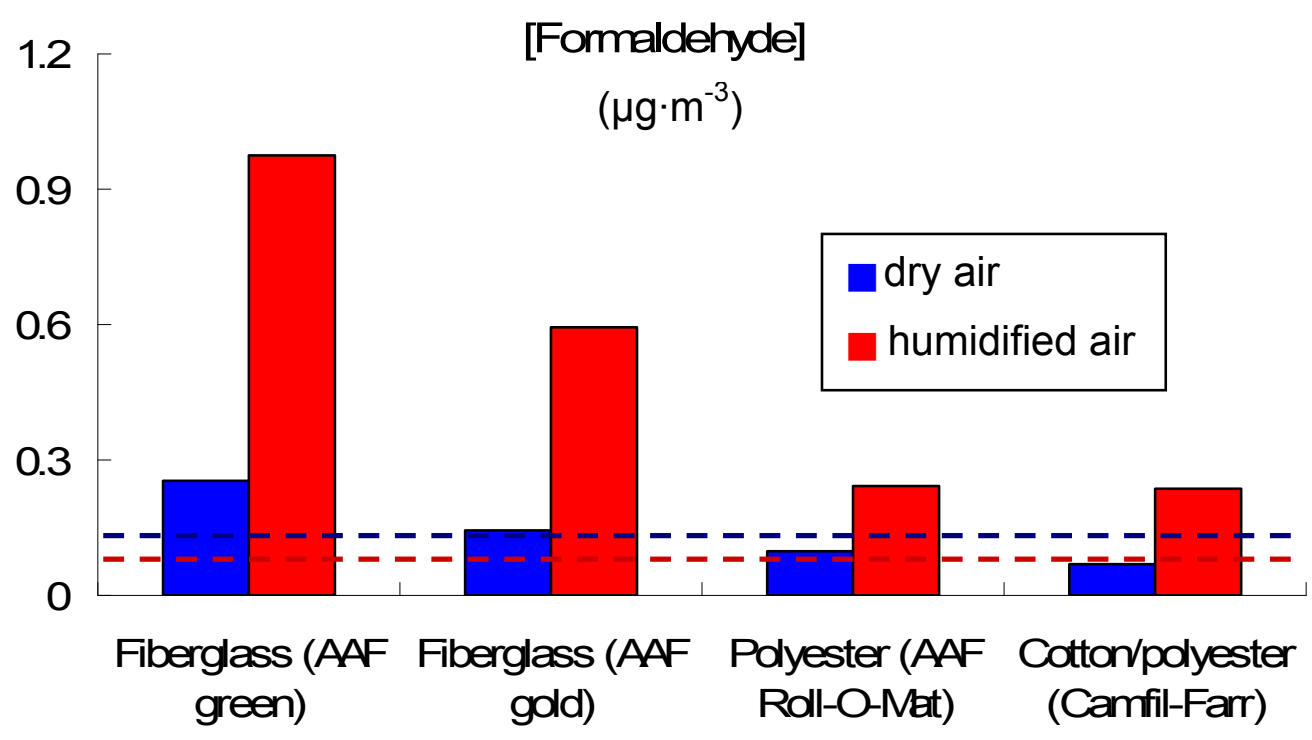

Figure 13: Formaldehyde concentrations measured downstream of unused filters. The limits of detection are indicated with dotted lines for dry air (blue) and humidified air (red). 


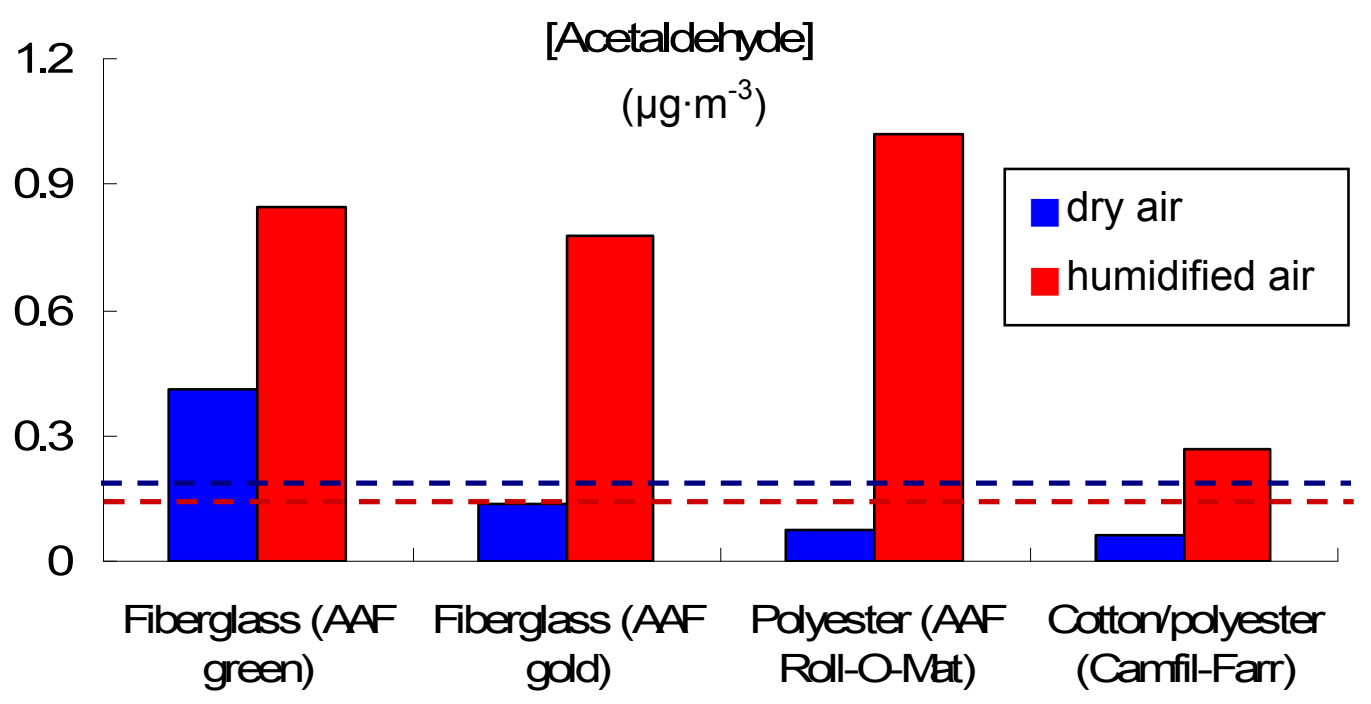

Figure 14: Acetaldehyde concentrations measured downstream of unused filters. The limits of detection are indicated with dotted lines for dry air (blue) and humidified air (red).

Results obtained for experiments using particle-laden filter media are presented in Figures 15 and 16 for formaldehyde (filters used in LBNL and the Port of Oakland, respectively) and for acetaldehyde in Figures 17 and 18 (filters used in LBNL and the Port of Oakland, respectively). The inlet ozone concentrations ranged from 140 to $197 \mathrm{ppb}$. Since the integrated samples were collected across the steady-state and initial periods of ozone breakthrough in each sample, aldehyde measurements corresponding to steady-state conditions only will likely be lower than those reported.

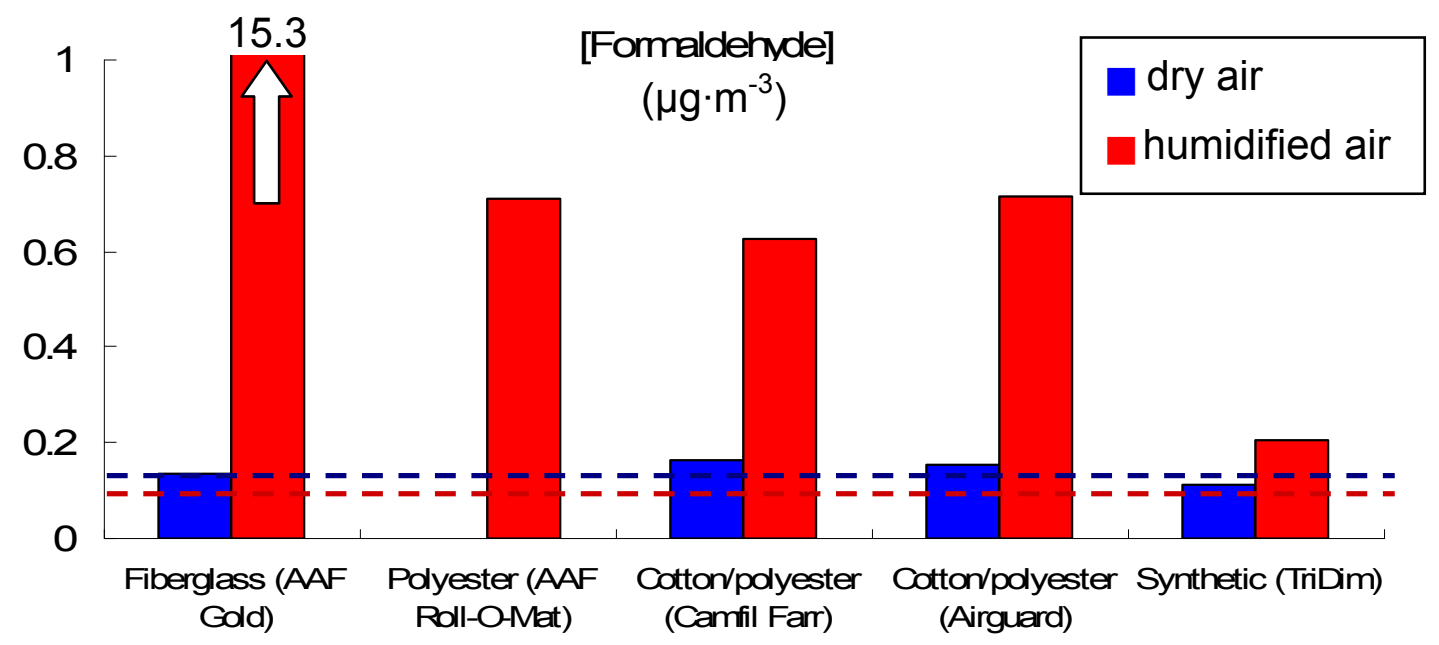

Figure 15: Formaldehyde concentrations measured downstream of filter used in LBNL. The limits of detection are indicated with dotted lines for dry air (blue) and humidified air (red). 


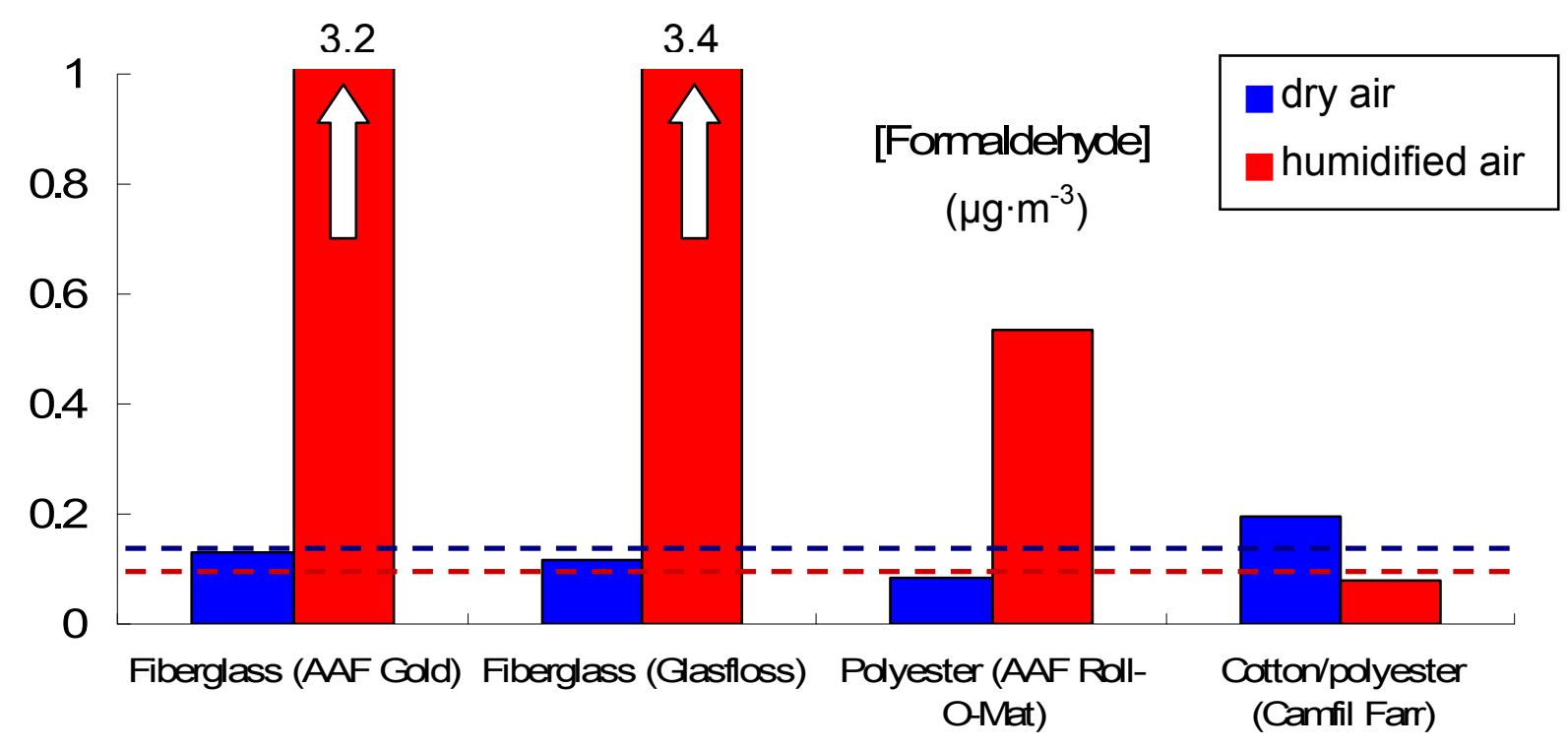

Figure 16: Formaldehyde concentrations measured downstream of filter used in the Port of Oakland. The limits of detection are indicated with dotted lines for dry air (blue) and humidified air (red).

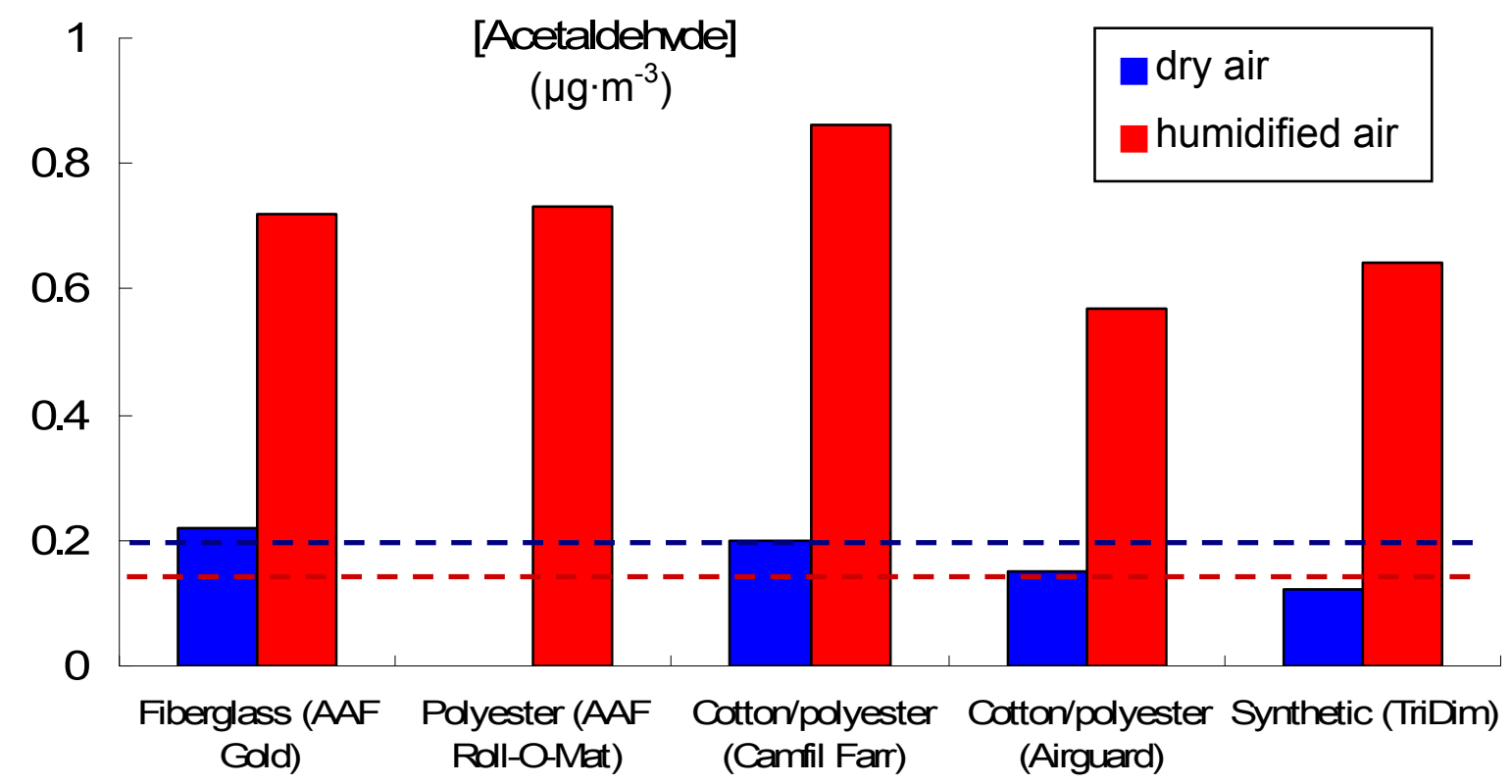

Figure 17: Acetaldehyde concentrations measured downstream of filter used in LBNL. The limits of detection are indicated with dotted lines for dry air (blue) and humidified air (red). 


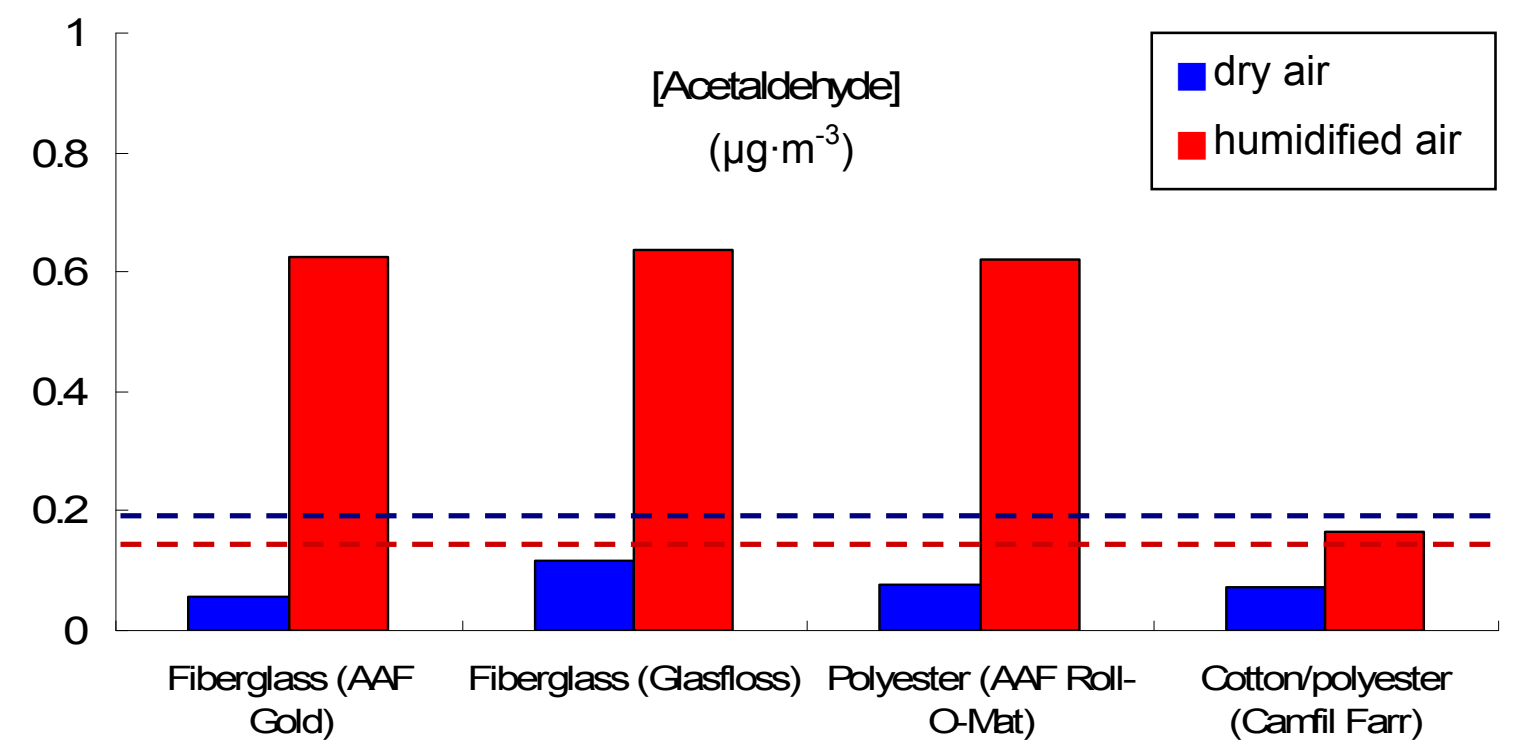

Figure 18: Acetaldehyde concentrations measured downstream of filter used in the Port of Oakland. The limits of detection are indicated with dotted lines for dry air (blue) and humidified air (red).

With particle-laden filters, we observed that experiments performed with dry air resulted in similarly low aldehyde concentrations as those recorded with unused filters, in most cases close to or below the limit of detection. Experiments in humidified air showed consistently higher outlet aldehyde concentrations, and particularly higher levels of formaldehyde, in the case of some fiberglass media.

In order to explore in more detail the high formaldehyde emissions from fiberglass HVAC media, we performed additional experiments in the absence of ozone to investigate if those very high emissions were due to ozone chemistry or to other chemical processes. We observed that maximum levels of formaldehyde were present with humidified air, even in the absence of ozone. High formaldehyde emissions were detected with unused filters, but were significantly higher in the case of used filters. Furthermore, in all the cases studied, for each combination of brand/model of filter and location in which the media was deployed, formaldehyde levels were higher in the absence of ozone, as compared with the same sample exposed to ozone. These results suggest the presence of a source that emits formaldehyde through hydrolysis. The presence of filter cake (in used filters) likely serves as a medium to condense and accumulate water vapor, thus catalyzing the hydrolysis process. Possible sources of formaldehyde in the presence of moisture are binders, impaction oils or other additives that may be present in or on the filter surface, or the accumulated filter contaminants. Results corresponding to the AAF Frontline Gold fiberglass media are shown in Figure 19, and those corresponding to the Glasfloss fiberglass media are shown in Figure 20. The AAF filters are coated with a thick layer of Viscosine (adhesive). The presence of impaction oils or other coatings in the Glasfloss filter is less evident, but cannot be ruled out as the Glasfloss vendor literature indicates the filter media has an adhesive coating. 


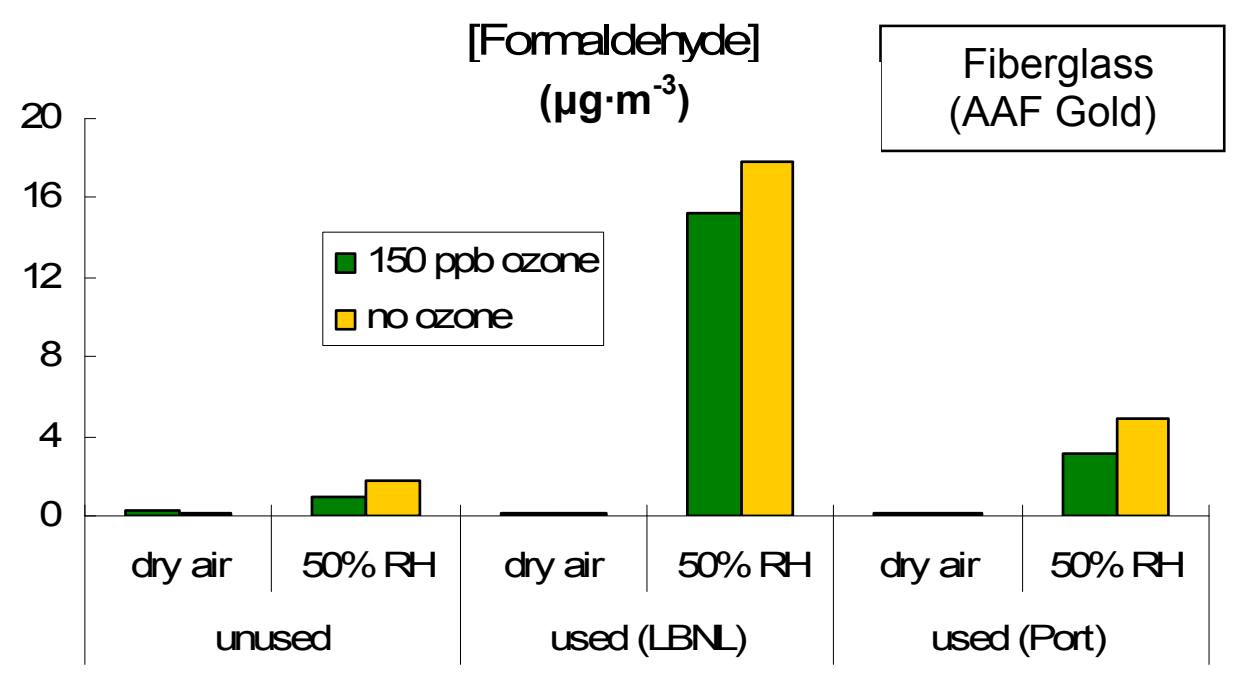

Figure 19: Outlet formaldehyde concentrations determined for the AAF Frontline Gold fiberglass filter under different conditions.

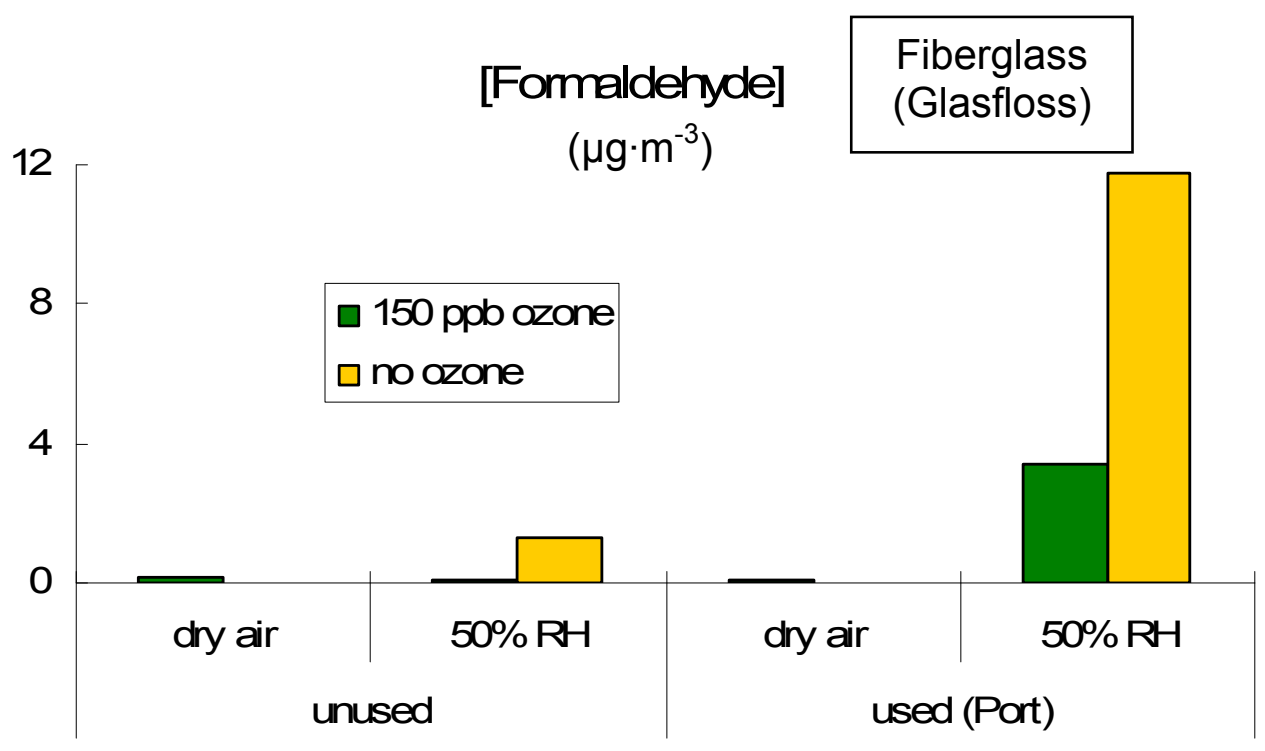

Figure 20: Outlet formaldehyde concentrations determined for the Glasfloss fiberglass filter under different conditions.

In addition, we performed the same analysis on two other types of HVAC media: polyester and cotton/polyester. The effects of ozone and humidity on 
formaldehyde emissions using these two filter media, shown in Figure 21, are different than described above for fiberglass media. In the case of polyester and cotton/polyester, overall formaldehyde emissions were significantly lower, and we did not observe emission of formaldehyde in the absence of ozone, even at $50 \%$ $\mathrm{RH}$. The AAF Roll-O-Mat Green media had a medium Viscosine application, while the Camfil Farr 30/50 media apparently had no treatment.

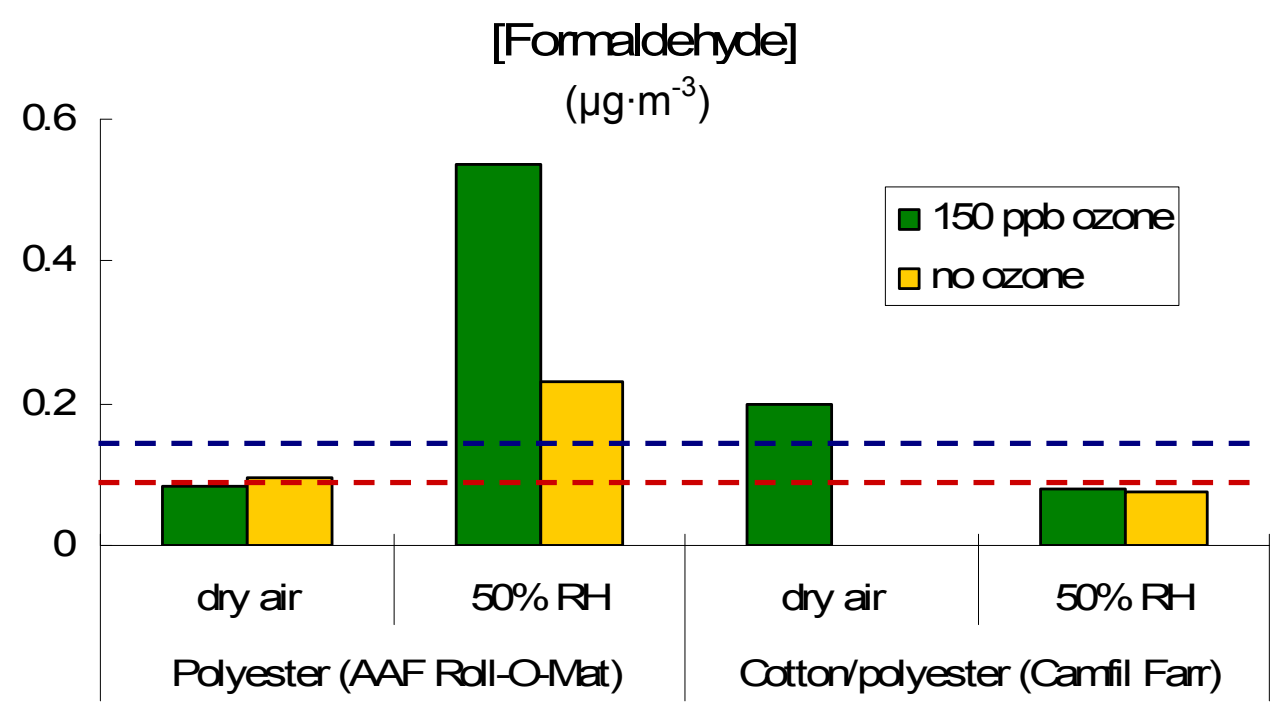

Figure 21: Formaldehyde concentrations measured downstream of polyester and cotton/polyester filters used in the Port of Oakland under various experimental conditions. 


\section{C.3. Effect of the face velocity}

Our experimental flow conditions corresponded to a face velocity of $0.013 \mathrm{~m} \mathrm{~s}^{-1}$, which is significantly lower than typical values existing in HVAC systems. Due to experimental limitations we could not access those high face velocity values without reduced accuracy in measurements of ozone losses and aldehyde emissions. However, we carried out two tests at a higher flow, to evaluate the approximate effect of flow velocity on both the ozone removal capacity and the emission of aldehydes. For these tests, we selected the AAF Gold filter used in LBNL's building 90, which exhibited one of the highest values of ozone removal and aldehyde emissions (although, as shown in the previous section, formaldehyde emissions do not completely originate in ozone chemistry). In all cases we used the same filter sample surface area of $1.7 \times 10^{-3} \mathrm{~m}^{2}$. The high flow condition was between 8 and 10 L. $\mathrm{min}^{-1}$, which is $\sim 1$ order of magnitude higher than the low airflow condition. The ozone removal curves for this filter at both face velocity conditions are shown in Figure 22 (for humidified air) and Figure 23 (dry air). In both cases, we represent the $\% \Delta\left[\mathrm{O}_{3}\right]$ value as a function of the air volume circulated through the filter.

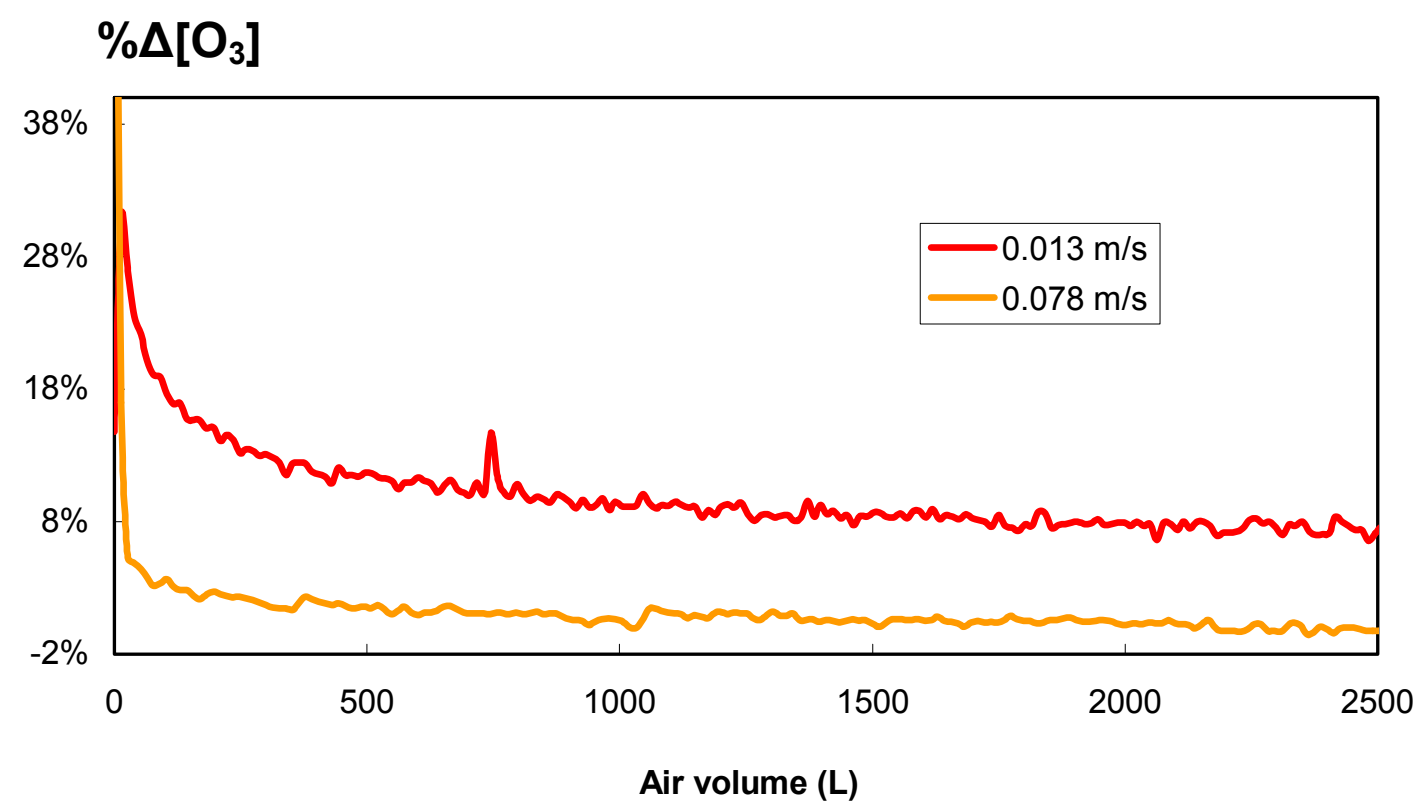

Figure 22: Ozone breakthrough curves for the used AAF Gold filter exposed to $150 \mathrm{ppb}$ ozone at two different flow conditions under humidified air. 


\section{$\% \Delta\left[\mathrm{O}_{3}\right]$}

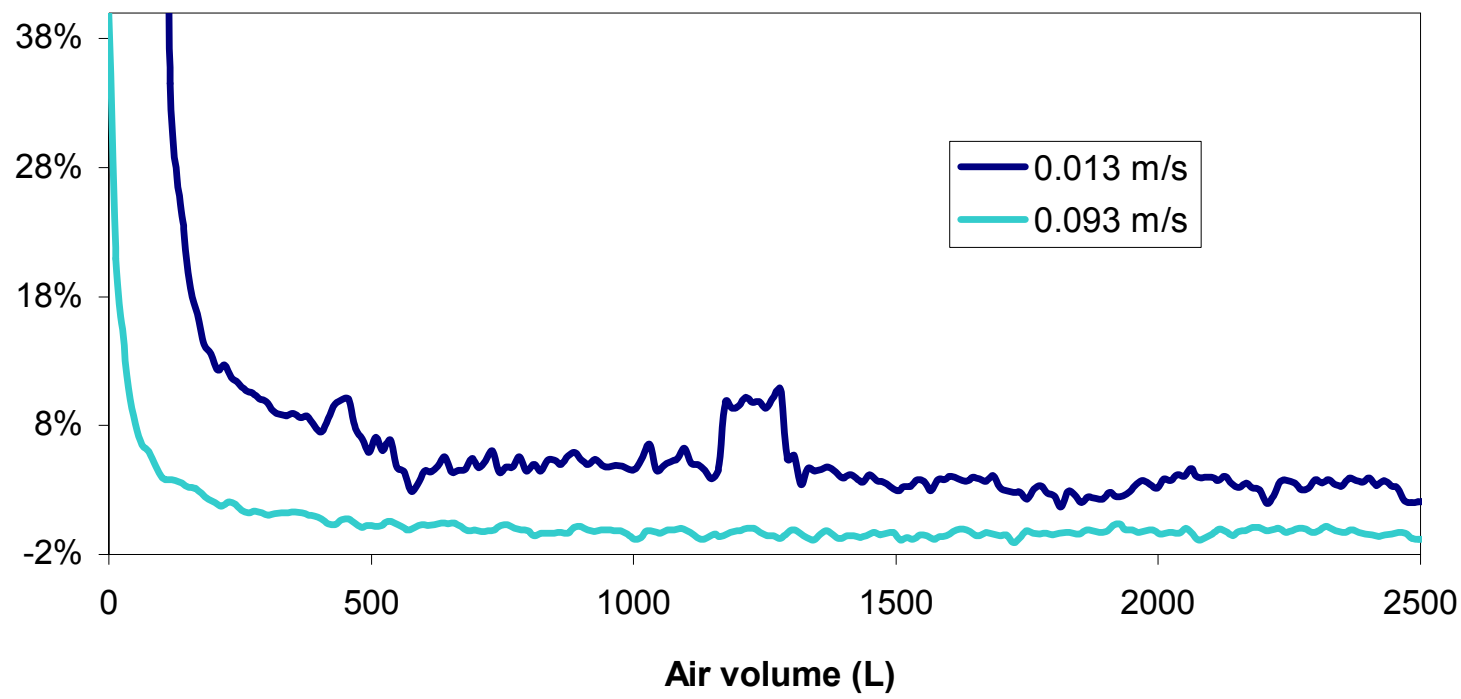

Figure 23: Ozone breakthrough curves for the used AAF Gold filter exposed to $150 \mathrm{ppb}$ ozone at two different flow conditions under dry air.

We observed that the ozone removed at a lower flow was significantly higher than at the higher flow, particularly in the case of humidified air. This indicates that the higher residence time corresponding to the low flow condition allowed for a more complete ozone reaction, and hence our ozone removal percentages determined at the low face velocity condition could be seen as an upper limit.

Table 4: Aldehyde downstream concentrations and emission rates (E) for $1.7 \times 10^{-3} \mathrm{~m}^{2}$ samples of AAF Gold filter media, at high and low airflow through the media in the presence of $150 \mathrm{ppb}$ ozone.

\begin{tabular}{|l|c|c|c|c|}
\hline \multirow{2}{*}{} & \multicolumn{2}{|c|}{ High Airflow } & \multicolumn{2}{c|}{ Low Airflow } \\
\cline { 2 - 5 } & Humidified & Dry & Humidified & Dry \\
\hline Airflow $\left(\mathbf{L ~} \mathbf{~ m i n}^{-1}\right)$ & 8.16 & 9.68 & 1.36 & 1.35 \\
\hline Face velocity $\left(\mathbf{m ~ s}^{-1}\right)$ & 0.078 & 0.093 & 0.013 & 0.013 \\
\hline Concentration $\left(\boldsymbol{\mu g ~ ~ ^ { - 3 } )}\right)$ & 1.96 & 0.014 & 15.3 & 0.13 \\
\hline Formaldehyde & 0.43 & 0.017 & 0.7 & 0.22 \\
\hline Acetaldehyde & 0.96 & 0.0080 & 1.26 & 0.0108 \\
\hline Emission rate $\left(\boldsymbol{\mu} \mathbf{g ~ h}^{-1}\right)$ & $6.59 \mathrm{E}-04$ & $2.69 \mathrm{E}-05$ & $1.92 \mathrm{E}-04$ & $4.75 \mathrm{E}-05$ \\
\hline Formaldehyde
\end{tabular}


In similar experiments, we collected DNPH samples downstream of the filters under the high flow condition, both for humidified and dry air. The results are reported in Table 4, and show that the emission rates (product of outlet concentration and flow rate) of both aldehydes were, in general, not significantly affected by the change in airflow, given the uncertainty in low aldehyde concentration measurements at high air flow rates. The data do suggest, however, an increase in acetaldehyde emission rate at high, relative to low, air flow rate with humidified air.

\section{C.4. Estimated Impacts on indoor aldehyde concentrations}

The expected increases in indoor aldehyde concentrations were estimated from the emission rates provided in Table 4 for the AAF Frontline Gold filter and from calculations of emission rates based on the data collected from tests of other used filters from the LBNL buildings at the low air flow rates with humidified air.. The steady-state indoor concentration increase $(\Delta \mathbf{C})$ was calculated from the equation

$$
\Delta C=\frac{E_{F A}}{Q_{F A}} f
$$

where $E_{F A}$ is the aldehyde emission rate per unit filter face area, $Q_{F A}$ is the outdoor air flow rate per unit filter face area and $f$ is a time units conversion factor. $E_{F A}$ is calculated as follows:

$$
E_{F A}=\frac{E}{0.0017 m^{2}} R
$$

where $E$ is the emission rate for a $0.0017 \mathrm{~m}^{2}$ sample of filter media from Table 4 and $R$ is the ratio of filter media area to filter face area. Examples values of $R$ include: unity for a pad filter, 3 to 4 for a $5 \mathrm{~cm}$ thick pleated filter, 6 to 7 for a 10 $\mathrm{cm}$ thick pleated filter, and 19 for a $56 \mathrm{~cm}$ deep bag filter. $Q_{F A}$ was estimated to equal $20 \%$ of the typical $2 \mathrm{~m}^{3} / \mathrm{s}$ of airflow per $1 \mathrm{~m}^{2}$ of filter face area, or $0.4 \mathrm{~m}^{3} / \mathrm{s}$ $\mathrm{m}^{2}$. We assume that $20 \%$ outdoor air is mixed with $80 \%$ recirculated air. 


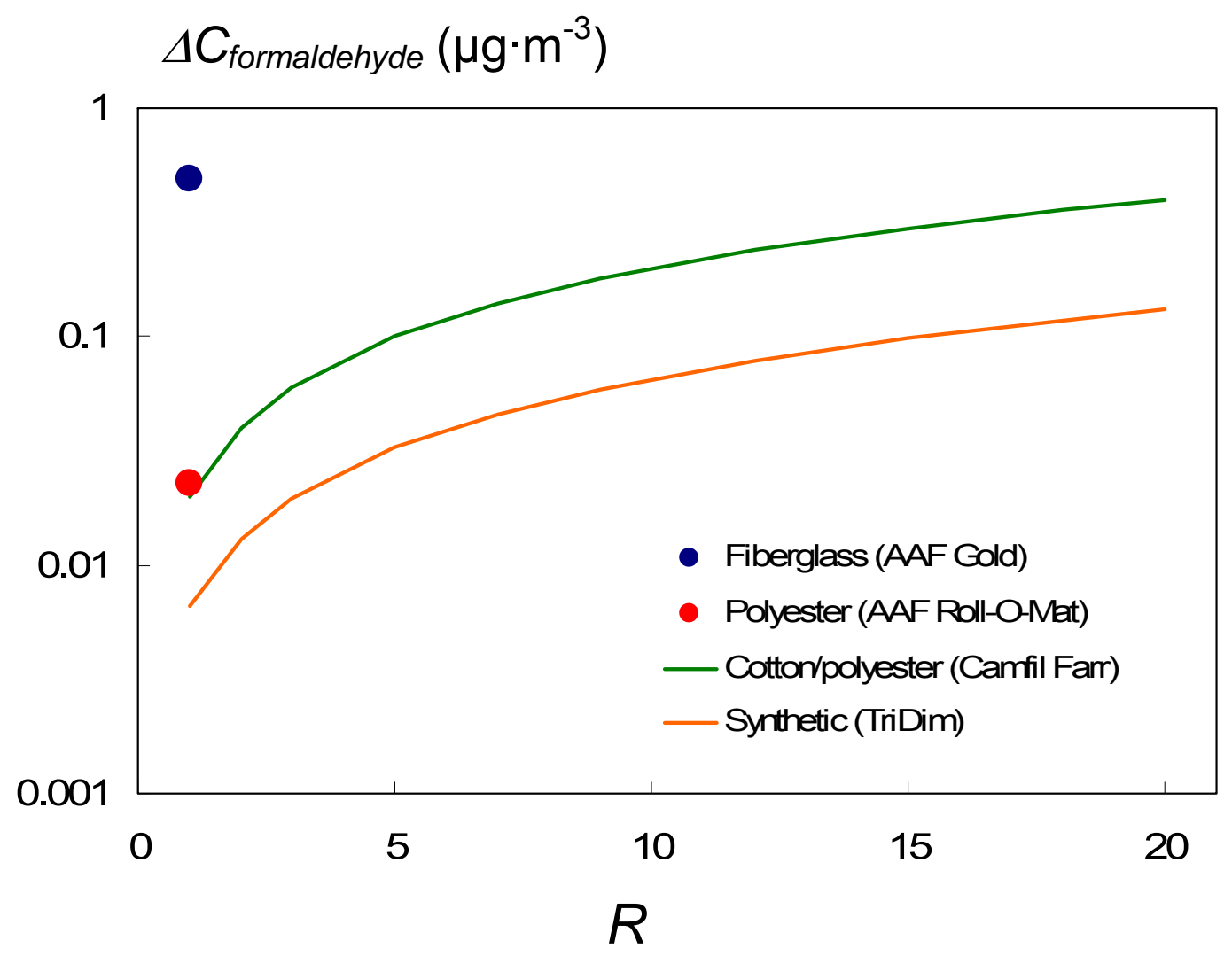

Figure 24: Estimated formaldehyde concentration increases at $50 \% \mathrm{RH}$, based on emission rates from filters used at LBNL, as a function of the ratio of filter media area to filter face area, $R$.

In Figure 24, we illustrate the expected indoor formaldehyde concentration increases for four of the studied filter media at $50 \% \mathrm{RH}$. The formaldehyde emission rates were obtained from the experiments with those filters used in LBNL buildings and exposed to ozone in humidified air at low airflows (approx $1.3 \mathrm{~L} / \mathrm{min}$ ). High formaldehyde emissions from the AAF Frontline Gold fiberglass filter media led to the highest predicted indoor concentration increases, approximately 0.5 for an $R$ value of 1 as indicated by the single blue point in Figure 24. This could be predicted to increase to approximately $10 \mu \mathrm{g} / \mathrm{m}^{3}$ if the results are extrapolated to an $R$ value of 19 . The calculations are for illustration purposes -- in practice the filters available on the market with a specific filter media type may have only a few values of $R$. Other filter samples, such as the polyester and cotton/polyester media, were estimated to increase the indoor formaldehyde concentration by 0.02 to $0.4 \mu \mathrm{g} / \mathrm{m}^{3}$, which are small increases relative to typical indoor formaldehyde concentrations. The synthetic filter media sample was estimated to increase the indoor formaldehyde concentration even less, ranging from less than $0.01 \mathrm{ug} / \mathrm{m} 3$ to slightly above $0.1 \mathrm{ug} / \mathrm{m} 3$. 


\section{Summary of Major Findings}

The results of this study confirm that there are significant reactions of ozone as air containing ozone flows through particle filters. The amount of ozone reacted is much higher when the filters are loaded with particles, particularly when the air is humidified. The amount of ozone reacted is not clearly related to the types of filter media, e.g., fiberglass versus synthetic. The ozone reaction rates were higher with filters made from American Air Filters, which have a Viscosine coating, suggesting ozone reaction with Viscosine. The nominal amount of Viscosine applied to different AAF Frontline fiberglass filters increases in the order Blue - Green - Gold. However, our results do not show clearly a net increase in ozone uptake that correlates with that order. Possibly, more than one type of impaction oil may be applied to each of these models, which makes it difficult to compare between those three products.

Aldehydes are produced as humidified air containing ozone passes through filters but also when humidified air without ozone passes through some of the filters (unused or particle-laden), suggesting that hydrolysis of filter binder or tackifier additives may at least partly explain the aldehyde emissions. Aldehyde emission rates were not clearly associated with filter media type. The estimated increases in indoor aldehyde concentrations are small relative to typical indoor aldehyde concentrations, except in a few cases in which formaldehyde emissions from filters were particularly higher and the results were extrapolated to other filter configurations.

To date, the results of this research do not help to explain why polyester or synthetic filters in environments with elevated ozone were statistically associated with increases in sick building syndrome symptoms from earlier analyses of the EPA's BASE study data.

Follow-on investigations include the study of low-level VOCs emitted by reactions of HVAC filters with ozone using Proton-Transfer Reaction Mass Spectrometry (PTR-MS), the effect of the presence of airborne VOCs during the reaction and the chemical characterization of filter cake before and after exposure to ozone. 